\section{LOWER SEMICONTINUITY, EXISTENCE AND REGULARITY THEOREMS FOR ELLIPTIC VARIATIONAL INTEGRALS OF MULTIPLE VALUED FUNCTIONS}

BY

PERTTI MATTILA

\begin{abstract}
Let $A$ be an open set in $\mathbf{R}^{\prime \prime}$ with compact smooth boundary, and let $\mathbf{Q}$ be the space of unordered $Q$ tuples of points of $\mathbf{R}^{\prime \prime}$. F. J. Almgren, Jr. has developed a theory for functions $f: A \rightarrow \mathbf{Q}$ and used them to prove regularity theorems for area minimizing integral currents. In particular, he has defined in a natural way the space $\because_{2}(A, \mathbf{Q})$ of functions $f: A \rightarrow \mathbf{Q}$ with square summable distributional partial derivatives and the Dirichlet integral $\operatorname{Dir}(f ; A)$ of such functions. In this paper we study more general constant coefficient quadratic integrals $\mathbf{G}(f: A)$ which are $Q$ elliptic in the sense that there is $c>0$ such that $\mathbf{G}(f: A) \geqslant c \operatorname{Dir}(f: A)$ for $f \in v_{2}(A ; \mathbf{Q})$ with zero boundary values. We prove a lower semicontinuity theorem which leads to the existence of a $\mathbf{G}$ minimizing function with given reasonable boundary values. In the case $m=2$ we also show that such a function is Hölder continuous and regular on an open dense set. In the case $m \geqslant 3$ the regularity problem remains open.
\end{abstract}

Introduction. In [A] F. J. Almgren, Jr. has developed a theory of functions defined on a bounded open subset $A$ of $\mathbf{R}^{m}$ with smooth boundary and taking values in the space $\mathbf{Q}$ of unordered $Q$ tuples of points of $\mathbf{R}^{n}$ (see [A2] for a summary). He studied a generalized Dirichlet integral for these functions and proved that for given $\mathbf{Q}$ valued boundary values in a Sobolev class there exists a $\mathbf{Q}$ valued Sobolev function minimizing such a Dirichlet integral and that the graph of this function outside an exceptional set of Hausdorff dimension at most $m-2$ is a real analytic submanifold of $\mathbf{R}^{m+n}$. This theory constituted a basic ingredient in Almgren's proof of the regularity theorem for $m$ dimensional area minimizing integral currents in $\mathbf{R}^{m+n}$, according to which the Hausdorff dimension of the singular set of such a current is at most $m-2$.

The Dirichlet integral is useful in the study of area minimizing currents because the corresponding integrand is the quadratic component of the area integrand in a suitable Taylor expansion. There are many interesting geometric integrands other than area, in particular the elliptic ones as defined in [A1, 1.6] or [F, 5.1.2], and it seems reasonable to investigate the variational calculus of $\mathbf{Q}$ valued functions in connection of their quadratic components. Consequently we shall consider quadratic polynomials $G$,

$$
G\left(p_{i}^{k}\right)=\sum q(i, j, k, l) p_{i}^{k} p_{j}^{l}
$$

Received by the editors September 21, 1982

1980 Mathematics Subject Classification. Primary 49F22.

$K e$. words and phrases. $\mathbf{Q}$ valued functions, $Q$ elliptic integrands, minimizing functions. 
on the space $P(n, m)$ of $n \times m$ matrices $\left(p_{i}^{h}\right)$. For any $f: A \rightarrow \mathbf{R}^{n}$ with square summable distributional partial derivatives $D_{i} f_{h}$ the $G$ integral of $f$ over $A$ is

$$
\mathbf{G}(f ; A)=\int_{A} \sum q(i, j, k, l) D_{i} f_{k} D_{i} f_{l} d \stackrel{i}{ }^{m},
$$

where $L^{m}$ is the Lebesgue measure. In a natural way $\mathbf{G}(f, A)$ can be defined also for functions $f: A \rightarrow \mathbf{Q}$. We say that $G$ is $Q$ elliptic if $\mathbf{G}(f ; A) \geqslant c \operatorname{Dir}(f ; A)$ for all Lipschitzian functions $f: A \rightarrow \mathbf{Q}$ with compact support where $c$ is a positive constant and $\operatorname{Dir}(f ; A)$ the Dirichlet integral of $f$. Then any quadratic component of a geometric elliptic integrand is $Q$ elliptic for every $Q$ (see $\S 3$ ).

In this paper we prove a lower semicontinuity theorem for $Q$ elliptic integrands (4.7)-(4.8), which leads to the existence of a $\mathbf{Q}$ valued $G$ minimizing function with given boundary values (5.1). The corresponding lower semicontinuity theorem for the Dirichlet integral in $[\mathbf{A}]$ is reduced to the result for single valued functions via a map $\boldsymbol{\xi}_{0}: \mathbf{Q} \rightarrow \mathbf{R}^{n Q}$ which arranges in a suitable way the coordinates of the $\mathbf{R}^{n}$ constituents of the elements of $\mathbf{Q}$. This map preserves the Dirichlet integral, but it does not preserve the general integrals $\mathbf{G}(f ; A)$ because of the presence of the mixed products $p_{i}^{k} p_{j}^{l}$, and therefore the same method to get the lower semicontinuity does not work.

In $\$ 6$ we prove that in the case $m=2$ the $\mathbf{G}$ minimizing $\mathbf{Q}$ valued functions are locally Hölder continuous, and we observe that in general continuity implies regularity on an open dense set. The continuity in the case $m \geqslant 3$ and the almost everywhere regularity in all cases remain as open problems.

It is well-known, see $[\mathbf{H}]$, that 1 ellipticity in the above sense is equivalent to the strong ellipticity condition

$$
\sum q(i, j, k, l) \xi_{i} \xi_{j} \eta_{k} \eta_{l}>0
$$

for $\left(\xi_{1}, \ldots, \xi_{m}\right) \neq 0$ and $\left(\eta_{1}, \ldots, \eta_{n}\right) \neq 0$. Since $Q$ ellipticity always implies 1 ellipticity, every $Q$ elliptic integrand is strongly elliptic. The converse is probably also true. In fact, it seems very likely that an algebraic result of Terpsta [T] could be used to prove that if $m=2$ or $n=2$ then every strongly elliptic integrand is a quadratic component of some convex, and hence elliptic, geometric integrand, and is therefore also $Q$ elliptic. However, Terpsta showed by an example that his result is false if $m \geqslant 3$ and $n \geqslant 3$, and this also implies that there are strongly elliptic integrands which are not quadratic components for convex geometric integrands. Still they might be $Q$ elliptic for all $Q$ 's, and even quadratic components for some nonconvex elliptic geometric integrand, if such things exist.

There are a great number of different definitions for ellipticity. In [A1] Almgren's definitions in the case of geometric integrands seem to depend on the coefficient group being used. In his thesis [S] V. Scheffer considered four notions of ellipticity for a $C^{1}$ integrand $A: R^{m} \times R^{n} \times P(n, m) \rightarrow P(n, m)$. Namely, in addition to the familiar notions of positive definiteness

$$
\left(D A_{a . b}(p)(z)\right) \cdot z \geqslant c|z|^{2} \quad \text { for } z \in P(n, m),
$$


where $c>0$ and $A_{a, h}(p)=A(a, b, p)$, and strong ellipticity

$$
\left(D A_{a, b}(p)(z)\right) \cdot z \geqslant c|z|^{2} \quad \text { for } z \in P(n, m) \text { with rank } z \leqslant 1,
$$

he introduced the following two new concepts:

$$
\int A(a, b, D \phi(x)+p) \cdot D \phi(x) d L^{m} x \geqslant c \int|D \phi(x)|^{2} d L^{m} x
$$

for $p \in P(n, m)$ and for every smooth $\phi: R^{m} \rightarrow R^{1}$ with compact support,

(4) $\left(\sum_{i=1}^{k} A\left(a, b, z_{i}^{\prime}\right)-\sum_{i=1}^{k} A\left(a, b, z_{i}\right)\right) \cdot\left(\sum_{i=1}^{k} z_{i}^{\prime}-\sum_{i=1}^{k} z_{i}\right) \geqslant c\left|\sum_{i=1}^{k} z_{i}^{\prime}-\sum_{i=1}^{k} z_{i}\right|$

whenever $z_{i}, z_{i}^{\prime} \in P(n, m)$ with rank $\left(\sum_{i=1}^{k} z_{i}^{\prime}-\sum_{i=1}^{k} z_{i}\right) \leqslant 1$.

In the linear case the definitions (2), (3) and (4) are all equivalent and strictly weaker than (1). In the general nonlinear case they all lead to different notions, and Scheffer proved that $(1) \Rightarrow(3) \Rightarrow(2)$ and $(4) \Rightarrow(3)$, the opposite implications being false, and also that neither (1) implies (4) nor (4) implies (1). As for the regularity of solutions of partial differential equations, these ellipticity conditions lead to different properties.

I want to thank Professor F. J. Almgren, Jr., for introducing me to this subject and for several helpful discussions.

1. Preliminaries. In our terminology and notation we follow $[\mathbf{A}]$ and $[\mathbf{F}]$. Throughout the whole paper $m, n$ and $Q$ will be positive integers.

1.1. The space $\mathbf{Q}$. The space of unordered $Q$ tuples of points in $\mathbf{R}^{n}$ is

$$
\mathbf{Q}=\mathbf{Q}\left(\mathbf{R}^{n}\right)=\left\{\sum_{i=1}^{Q}\left[\left[y^{i}\right]\right]: y^{1}, \ldots, y^{Q} \in \mathbf{R}^{n}\right\}
$$

where $\left[\left[y^{i}\right]\right]$ is the Dirac measure at $y^{i} . \mathbf{Q}$ is equipped with the metric $\mathcal{G}$;

$$
\mathcal{G}\left(\sum_{i=1}^{Q}\left[\left[y^{i}\right]\right], \sum_{i=1}^{Q}\left[\left[z^{i}\right]\right]\right)=\inf _{\sigma}\left(\sum_{i=1}^{Q}\left|y^{i}-z^{\sigma(i)}\right|^{2}\right)^{1 / 2}
$$

where $\sigma$ runs through all the permutations of $\{1, \ldots, Q\}[\mathbf{A}, 1.1(2)]$.

There are a bi-Lipschitzian homeomorphism $\xi: \mathbf{Q} \rightarrow \mathbf{Q}^{*} \subset \mathbf{R}^{P Q}$ where $P$ is a positive integer, and a Lipschitzian retraction $\rho: \mathbf{R}^{P Q} \rightarrow \mathbf{Q}^{*}$ with $\rho \mid \mathbf{Q}^{*}=\mathbf{1}_{\mathbf{Q}^{*}}$ $[A, 1.1(6),(8)]$. The map $\xi$ is defined by means of $P$ suitably chosen orthogonal projections $\pi_{1}, \ldots, \pi_{P}: \mathbf{R}^{n} \rightarrow \mathbf{R}$ by the condition that $\xi\left(\sum_{i}\left[\left[y^{i}\right]\right]\right)=z$ if and only if for each $k=1, \ldots, n$ there is a permutation $\sigma_{k}$ of $\{1, \ldots, Q\}$ such that $y_{k}^{\sigma_{k}(1)} \leqslant y_{k}^{\sigma_{k}(2)} \leqslant$ $\cdots \leqslant y_{k}^{\sigma_{k}(Q)}$ and $z_{(k-1) Q+i}=y_{k}^{\sigma_{k}(i)}$ for $i=1, \ldots, Q$. The first projections are taken to be the usual coordinate functions $\pi_{i}(x)=x_{i}$ for $x \in \mathbf{R}^{n}$ and $i=1, \ldots, n$.

1.2. Affine approximations. The set of all affine maps $\mathbf{R}^{m} \rightarrow \mathbf{R}^{n}$ is denoted by $A(n, m)$. If $h \in A(n, m)$, we put

$$
|h|=\left(\sum_{i, k}\left(D_{i} h_{k}\right)^{2}\right)^{1 / 2} \in \mathbf{R}
$$


where $D_{i} h_{k}$ also denotes the constant value of $D_{i} h_{k}$. A function $h: \mathbf{R}^{m} \rightarrow \mathbf{Q}$ is called affine if there are $h^{1}, \ldots, h^{Q} \in A(n, m)$ such that $h=\sum_{i=1}^{Q}\left[\left[h^{i}\right]\right]$, i.e., $h(a)=$ $\sum_{i=1}^{Q}\left[\left[h^{i}(a)\right]\right]$ for $a \in \mathbf{R}^{m}$. Then we set $|h|=\left(\sum_{i=1}^{Q}\left|h^{i}\right|^{2}\right)^{1 / 2}$.

If $A \subset \mathbf{R}^{m}$ is open and $a \in A$, a function $f: A \rightarrow \mathbf{Q}$ is said to be approximately affinely approximable at $a$ if there is an affine function $h: \mathbf{R}^{m} \rightarrow \mathbf{Q}$ such that

$$
\underset{x \rightarrow a}{\operatorname{ap} \lim }|x-a|^{-1} \Theta(f(x), h(x))=0 .
$$

Such a function $h$ is uniquely determined and denoted by ap $A f(a)$. If $f$ is approximately affinely approximable at $a, \operatorname{ap} A f(a)=\sum_{i=1}^{Q}\left[\left[h^{i}\right]\right]$ and for all $i, j$ either $h^{i}=h^{j}$ or $h^{i}(a) \neq h^{j}(a)$, then $f$ is said to be strongly approximately affinely approximable at $a$. This is true if $\boldsymbol{\xi} \circ f$ is approximately differentiable at $a$, and then (see $[\mathbf{A}, 1.4(3)])$

$$
(\operatorname{Lip} \xi)^{-1}|\operatorname{ap} D(\xi \circ f)(a)| \leqslant|\operatorname{ap} A f(a)| \leqslant|\operatorname{ap} D(\xi \circ f)(a)| .
$$

Clearly, if $Q=1, \operatorname{ap} A f(a)=$ ap $D f(a)+f(a)$.

1.3. The space ${ }_{2}(A, \mathbf{Q})$. Suppose $A \subset \mathbf{R}^{m}$ is open.

(1) If $k$ is a positive integer, we denote by $\mathrm{g}_{2}\left(A, \mathbf{R}^{k}\right)$ the Sobolev space of $\mathbf{R}^{k}$ valued functions on $A$ which together with their first order distributional partial derivatives are $\mathbb{L}^{m}$ square summable over $A$. A function $f \in \mathrm{Q}_{2}\left(A, \mathbf{R}^{k}\right)$ is said to be strictly defined if $f(x)=y$ whenever $x \in A, y \in \mathbf{R}^{k}$ and

$$
\lim _{r ! 0} r^{-m} \int_{B(x, r)}|f(z)-y| d \mathbb{L}^{m} z=0
$$

Any $f \in \mathrm{S}_{2}\left(A, \mathbf{R}^{k}\right)$ agrees $\mathbb{L}^{m}$ almost everywhere on $A$ with a strictly defined $g \in \mathrm{N}_{2}\left(A, \mathbf{R}^{k}\right)$. If $f \in \mathrm{S}_{2}\left(A, \mathbf{R}^{k}\right)$ is strictly defined, then

$$
\lim _{r \downarrow 0} r^{-m} \int_{B(x, r)}|f(z)-f(x)| d \check{L}^{m} z=0
$$

for $\mathfrak{K}^{m-1}$ almost all $x \in A$ (see [A, A.1.2(3)]). Here $\mathfrak{K}^{m-1}$ is the $m-1$ dimensional Hausdorff measure. For any such point $x \in A$ and for any open set $B \subset A$ for which $x \in \partial B$, it follows that

$$
f(x)=\lim _{r \downarrow 0} \mathbb{L}^{m}(B(x, r) \cap B)^{-1} \int_{B(x, r) \cap B} f d \mathbb{L}^{m} .
$$

(2) We say that a function $f: A \rightarrow \mathbf{R}^{k}$ is ACL (absolutely continuous on lines) if whenever $R$ is a cube contained in $A$ with sides parallel to the coordinate axis and $\pi$ is an orthogonal projection of $R$ onto an $m-1$ dimensional face $S$ of $R, f \mid \pi^{-1}\{y\}$ is absolutely continuous for $\mathfrak{K}^{m-1}$ almost all $y \in S$. Any strictly defined function in $\mathrm{O}_{2}\left(A, \mathbf{R}^{k}\right)$ is ACL [M, 3.1.8]. Conversely, if $f$ is $\mathrm{ACL}$ and $f$ and its first order partial derivatives are $L^{m}$ square summable over $A$, then $f \in \mathcal{O}_{2}\left(A, \mathbf{R}^{k}\right)$.

(3) The space $Q_{2}(A, \mathbf{Q})$ consists of those functions $f: A \rightarrow \mathbf{Q}$ for which $\boldsymbol{\xi} \circ f \in$ $\mathrm{S}_{2}\left(A, \mathbf{R}^{P Q}\right)$. We say that $f$ is strictly defined if $\boldsymbol{\xi} \circ f$ is strictly defined. If $f, g \in$ $\mathrm{O}_{2}(A, \mathbf{Q})$, we write $f=g$ if $f(x)=g(x)$ for $E^{m}$ almost all $x \in A$.

(4) The space $\partial \mathcal{O}_{2}(\partial A, \mathbf{Q})$ will be the set of those functions $g: \partial A \rightarrow \mathbf{Q}$ for which there is a strictly defined $f \in \mathcal{O}_{2}\left(\mathbf{R}^{m}, \mathbf{Q}\right)$ such that $f(x)=g(x)$ for $\mathcal{K}^{m-1}$ almost all 
$x \in \partial A$ (cf. [A, A.1.2(7) and 2.1(2)]). If $g, h \in \partial \mathrm{P}_{2}(\partial A, \mathbf{Q})$, we write $g=h$ if $g(x)=$ $h(x)$ for $\mathcal{H}^{-m-1}$ almost all $x \in \partial A$. If $B$ is an open subset of $A$ and $g \in \partial \mathscr{Q}_{2}(\partial B, \mathbf{Q})$, we say that $f$ has boundary values $g$ if there is $h \in \mathscr{q}_{2}\left(\mathbf{R}^{m}, \mathbf{Q}\right)$ which is strictly defined such that $h|B=f| B$ and $h \mid \partial B=g$ [A, A.1.1(7)]. We then write $f_{\mid \partial B}=g$. If $f$ is strictly defined, $g$ agrees with $f \mathfrak{K}^{m-1}$ almost everywhere on $A \cap \partial B$. By writing $f_{\mid \partial B} \in \partial \mathcal{Y}_{2}(\partial B, \mathbf{Q})$ we mean that there is $g \in \partial \mathcal{O}_{2}(\partial B, \mathbf{Q})$ such that $f_{\mid \partial B}=g$, and by $f_{\mid \partial B}=h_{\mid \partial B} \in \partial \mathcal{O}_{2}(\partial B, \mathbf{Q})$ we mean that there is $g \in \partial \mathcal{Q}_{2}(\partial B, \mathbf{Q})$ such that $f_{\mid \partial B}=g$ and $h_{\mid \partial B}=g$.

(5) Suppose $\mathfrak{H}^{m-1}(\partial A)<\infty$. If $f, g \in \mathscr{Q}_{2}\left(\mathbf{R}^{m}, \mathbf{Q}\right)$ and $f_{\mid \partial A}=g_{\mid \partial A} \in \partial \vartheta_{2}(\partial A, \mathbf{Q})$, then $h=f|A \cup g| \mathbf{R}^{m} \sim A \in \mathrm{O}_{2}\left(\mathbf{R}^{m}, \mathbf{Q}\right)$ (cf. [A, A.1.1(9)]). One way to verify this is to show that if $f$ and $g$ are strictly defined, and hence ACL, then $h$ is ACL. This clearly suffices, and it follows from the facts that if $\pi$ is the orthogonal projection of $\mathbf{R}^{m}$ onto a hyperplane $V$, then for $\mathfrak{H}^{m^{-1}}$ almost all $y \in V$ the set $\partial A \cap \pi^{-1}\{y\}$ is finite and the set $\partial A \cap \pi^{-1}\{y\} \cap\{x: f(x) \neq g(x)\}$ is empty [F, 2.10.11].

(6) Suppose $f \in \mathcal{O}_{2}(A, \mathbf{Q})$ and $B$ is an $\varrho^{m}$ measurable set with $\varrho^{m}(B \sim A)=0$. Then $f$ is approximately affinely approximable $\mathcal{L}^{m}$ almost everywhere on $B$ and the Dirichlet integral of $f$ over $B$ is

$$
\operatorname{Dir}(f ; B)=\int_{B}|\operatorname{ap} A f(x)|^{2} d L^{m} x
$$

If $g: \mathbf{Q} \rightarrow \mathbf{R}^{k}$ is Lipschitzian, then $g \circ f \in \mathscr{Y}_{2}\left(A, \mathbf{R}^{k}\right)$ and $\mid$ ap $D(g \circ f)(x) \mid$ $\leqslant \operatorname{Lip} g|\operatorname{ap} A f(x)|$ for $E^{m}$ almost all $x \in A$, as one can show by [A, 1.4(1)], whence $\operatorname{Dir}(g \circ f ; B) \leqslant(\operatorname{Lip} g)^{2} \operatorname{Dir}(f ; B)$.

(7) If $V$ is a hyperplane in $\mathbf{R}^{m}, B \subset V$ is $\mathscr{H}^{m-1}$ measurable, $\varphi: \mathbf{R}^{m-1} \rightarrow V$ is an isometry, $g: B \rightarrow \mathbf{Q}$ and $g \circ \varphi$ is approximately affinely approximable $\mathcal{L}^{m-1}$ almost everywhere on $\varphi^{-1}(B)$, we denote

$$
\operatorname{dir}(g ; B)=\operatorname{Dir}\left(g \circ \varphi ; \varphi^{-1}(B)\right)
$$

(cf. [A, 1.1(6)]). If $f \in \mathscr{Y}_{2}\left(\mathbf{R}^{m}, \mathbf{Q}\right)$ is strictly defined, $f \mid B=g$ and $f$ is approximately affinely approximable $\mathcal{H}^{m-1}$ almost everywhere on $B$, then

$$
\operatorname{dir}(g ; B) \leqslant \int_{B}|\operatorname{ap} A f(x)|^{2} d \mathcal{H}^{m-1} x .
$$

If $R$ is a rectangle in $\mathbf{R}^{m}$ and $g: \partial R \rightarrow \mathbf{Q}$, we set

$$
\operatorname{dir}(g ; \partial R)=\sum\{\operatorname{dir}(g ; S): S \text { an } m-1 \text { face of } R\}
$$

in case the right-hand side is defined.

(8) Lemma. Suppose $f, f_{i} \in \mathcal{O}_{2}(A, \mathbf{Q}), i=1,2, \ldots$, are strictly defined, $\operatorname{Dir}(f, A)$, $\operatorname{Dir}\left(f_{i} ; A\right)<M<\infty$ for $i=1,2, \ldots, \lim _{i \rightarrow x} \int_{A} \mathcal{G}\left(f, f_{i}\right)^{2} d \mathcal{L}^{m}=0, E \subset A$ is $\left(\mathcal{H}^{m-1}, m-1\right)$ rectifiable $\mathcal{H}^{m-1}$ measurable set $[\mathbf{F}, 3.2 .14], g: E \rightarrow \mathbf{Q}$ and $f_{i}(x)=$ $g(x)$ for $x \in E, i=1,2, \ldots$ Then $f(x)=g(x)$ for $\mathcal{H}^{m-1}$ almost all $x \in E$.

Proof. It is sufficient to prove the lemma for real-valued functions $f, f_{i} \in$ $\mathcal{\vartheta}_{2}(A, \mathbf{R})$. Suppose the lemma is false. Then there are $\varepsilon>0$, a cube $R \subset A$ with sides parallel to the coordinate axis and a closed set $F \subset E \cap R$ such that $\mathcal{H}^{m-1}(F)>0$ 
and $|f(x)-g(x)| \geqslant \varepsilon$ for $x \in F$. Since $F$ is $\left(\mathcal{K}^{m-1}, m-1\right)$ rectifiable, there is an $m-1$ dimensional face $S$ of $R$ parallel to $\left\{x \in \mathbf{R}^{m}: x_{j}=0\right\}$ for some $j$ such that $\mathfrak{H}^{m-1}(\pi F)>0$ where $\pi: R \rightarrow S$ is the orthogonal projection [F, 3.2.27]. Let $0<\delta<$ $\varepsilon^{2} \cdot \mathcal{H}^{m-1}(\pi F) /(18 M)$,

$$
\begin{gathered}
B=\left\{y \in R: \operatorname{dist}\left(y, F \cap \pi^{-1}\{\pi(y)\}\right)<\delta\right\}, \\
B_{i}=\left\{y \in B:\left|f(y)-f_{i}(y)\right|<\varepsilon / 3\right\}, \quad i=1,2, \ldots .
\end{gathered}
$$

Then $\pi B=\pi F$ and $\mathscr{L}^{\prime \prime}\left(B \sim B_{i}\right) \rightarrow 0$, which implies $\mathfrak{K}^{m-1}\left(\pi B_{i}\right) \rightarrow \mathfrak{H}^{m-1}(\pi B)=$ $\mathcal{H}^{\prime \prime}(\pi F)$, because

$$
\begin{aligned}
& \mathbb{L}^{\prime \prime}\left(B \sim B_{i}\right)=\int_{S} \mathfrak{K}^{-1}\left(\left(B \sim B_{i}\right) \cap \pi^{-1}\{y\}\right) d \mathfrak{H}^{m-1} y \\
& \geqslant \int_{\pi B \sim \pi B_{i}} \mathfrak{K}^{-1}\left(B \cap \pi^{-1}\{y\}\right) d \cdot \mathcal{H}^{m-1} y \geqslant \delta \mathcal{H}^{m-1}\left(\pi B \sim \pi B_{i}\right) .
\end{aligned}
$$

Suppose $z \in \pi B_{i}$ is such that $f \mid \pi^{-1}\{z\}$ and $f_{i} \mid \pi^{-1}\{z\}$ are absolutely continuous, which is true for $: x^{m-1}$ almost all $z \in \pi B_{i}$. There are $y \in B_{i} \cap \pi^{-1}\{z\}$ and $x \in F \cap$ $\pi^{-1}\{z\}$ such that $|x-y|<\delta$. Then either $|f(x)-f(y)| \geqslant \varepsilon / 3$ or $\left|f_{i}(x)-f_{i}(y)\right| \geqslant$ $\varepsilon / 3$, because $y \in B_{i},|f(x)-g(x)| \geqslant \varepsilon$ and $f_{i}(x)=g(x)$. By the absolute continuity and Hölder's inequality the first alternative implies

$$
\int_{\pi^{-1}\{=\}}\left(D_{j} f\right)^{2} d \cdot \mathcal{H}^{1} \geqslant(\varepsilon / 3)^{2} / \delta
$$

and the second

$$
\int_{\pi^{-1}\{=\}}\left(D_{j} f_{i}\right)^{2} d \cdot \dot{H}^{1} \geqslant(\varepsilon / 3)^{2} / \delta
$$

Then Fubini's theorem gives

$$
\begin{aligned}
2 M & \geqslant \operatorname{Dir}(f ; R)+\operatorname{Dir}\left(f_{i} ; R\right) \\
& \geqslant \int_{\pi B_{i}}\left[\int_{\pi^{-1}\{z\}}\left(D_{j} f\right)^{2} d \cdot \mathcal{K}^{-1}+\int_{\pi^{-1}\{z\}}\left(D_{j} f_{i}\right)^{2} d \mathcal{K}^{-1}\right] d \mathcal{H}^{-m-1} z \\
& \geqslant\left(\varepsilon^{2} / 9 \delta\right): \mathcal{H}^{-m-1}\left(\pi B_{i}\right) .
\end{aligned}
$$

Letting $i \rightarrow \infty$ we get a contradiction $\delta \geqslant \varepsilon^{2 \cdot \pi^{m-1}}(\pi F) /(18 M)$.

1.4. Quadratic integrands. Let $P(n, m)$ be the set of real $n \times m$ matrices $\left(p_{i}^{k}\right)$, $i=1, \ldots, m, k=1, \ldots, n$. For any function $G: P(n, m) \rightarrow \mathbf{R}$ we denote

$$
\begin{gathered}
G h=G\left(D_{i} h_{k}\right) \quad \text { for } h \in A(n, m), \\
G h=\sum_{i=1}^{Q} G h^{i} \quad \text { for } h=\sum_{i=1}^{Q}\left[\left[h^{i}\right]\right]: \mathbf{R}^{m} \rightarrow \mathbf{Q}, \quad h^{i} \in A(n, m) .
\end{gathered}
$$

If $f: B \rightarrow \mathbf{Q}, B \subset \mathbf{R}^{m}$, we set $G f(a)=G$ ap $A f(a)$ whenever ap $A f(a)$ exists, and $\mathbf{G}(f ; B)=\int_{B} G f(x) d L^{m} x$ whenever $\int_{B} G f(x) d L^{m} x$ exists. In the rest of this paper we shall assume that $G$ is a quadratic constant coefficient integrand given by

$$
G\left(p_{i}^{k}\right)=\sum q(i, j, k, l) p_{i}^{k} p_{j}^{l}
$$


where $q(i, j, k, l), i, j=1, \ldots, m, k, l=1, \ldots, n$, are real numbers which satisfy

$$
q(i, j, k, l)=q(j, i, l, k) \text {. }
$$

Clearly this is no restriction, because the original coefficients $q(i, j, k, l)$ and $q(j, i, l, k)$ could be replaced by $(q(i, j, k, l)+q(j, i, l, k)) / 2$ without affecting the values of $G$.

The integrand leading to the Dirichlet integral is $\left(p_{i}^{k}\right) \mapsto|p|^{2}=\Sigma\left(p_{i}^{k}\right)^{2}$. We shall fix for the rest of the paper a constant $C_{0}$ such that for any $n \times m$ matrix $p$, $|G p| \leqslant C_{0}|p|^{2}$. Then if $f \in \mathrm{q}_{2}(A, \mathbf{Q})$ and $B$ is $\mathfrak{L}^{m}$ measurable with $\mathfrak{L}^{m}(B \sim A)=0$,

$$
|\mathbf{G}(f ; B)| \leqslant C_{0} \operatorname{Dir}(f ; B) \text {. }
$$

We say that $G$ is $Q$ elliptic if there is $c>0$ such that

$$
\mathbf{G}\left(f ; \mathbf{R}^{m}\right) \geqslant c \operatorname{Dir}\left(f ; \mathbf{R}^{m}\right)
$$

whenever $f: \mathbf{R}^{m} \rightarrow \mathbf{Q}$ is Lipschitzian with compact support, that is, $\boldsymbol{\xi} \circ f$ has compact support. If this holds with $c=0, G$ is called $Q$ semielliptic. In both cases $c$ is called a $Q$ ellipticity bound of $G$. It follows from an approximation theorem of $\mathrm{J}$. H. Michael (see [A, A.1.2(2)]), that in (1) Lipschitzian functions can be replaced by functions in $\mathrm{N}_{2}\left(\mathbf{R}^{m}, \mathbf{Q}\right)$ with compact support.

As remarked in the Introduction, I do not know whether, in fact, $Q$ ellipticity would reduce to the usual notion of strong ellipticity.

1.5. Intervals in $\mathbf{R}^{m}$. By an interval in $\mathbf{R}^{m}$ we mean a nondegenerate rectangle with sides parallel to the coordinate axis. We shall denote by $R$ the set of all open intervals in $\mathbf{R}^{m}$ for which the ratios of the side-lengths are all strictly less than 2 . We also denote by $R$ the corresponding $\mathcal{L}^{m}$ Vitali relation [F, 2.8.16] consisting of all pairs $(x, R)$ such that $R \in R$ and $x$ is the centre of $R$. It is well known [F, 2.9.8] that for any locally $\mathcal{L}^{m}$ summable function $f: \mathbf{R}^{m} \rightarrow \mathbf{R}$

$$
\text { ( R ) } \lim _{R \rightarrow x} \varrho^{m}(R)^{-1} \int_{R} f d \varrho^{m}=f(x)
$$

for $\mathbb{L}^{m}$ almost all $x \in \mathbf{R}^{m}$.

We shall use the following notation. Given $0<t<\infty$ and an open interval $R$ with centre $x$ and side-lengths $d_{1}, \ldots, d_{m}, t R$ will stand for the open interval with centre $x$ and side-lengths $t d_{1}, \ldots, t d_{m}$. If $f: \mathbf{R}^{m} \rightarrow \mathbf{R}$ is $\varrho^{m}$ locally summable and $1<t<\infty$, then it follows readily from (2) that

$$
\text { ( } R) \lim _{R \rightarrow x} \varrho^{m}(t R \sim R)^{-1} \int_{t R \sim R} f d \varrho^{m}=f(x)
$$

for $\mathbb{L}^{m}$ almost all $x \in \mathbf{R}^{m}$.

2. Some lemmas for $\mathbf{Q}$ valued functions.

2.1. Definition. We define $S_{k}: \mathbf{Q} \rightarrow \mathbf{R}, k=1, \ldots, n, b_{\vee}$ '

$$
S_{k}\left(\sum_{i=1}^{Q}\left[\left[y^{i}\right]\right]\right)=\sum_{i=1}^{Q} y_{k}^{i} \text {. }
$$


2.2. Lemma. Suppose $A \subset \mathbf{R}^{m}$ is open, $f: A \rightarrow \mathbf{Q}, a \in A$ and $\boldsymbol{\xi} \circ f$ is approximately differentiable at $a$.

(1) There is a neighborhood $U$ of a such that $\xi \circ$ ap $A f(a)|U=\operatorname{ap} A(\xi \circ f)(a)| U$.

(2) For $k=1, \ldots, n, S_{k} \circ f=\sum_{i=1}^{Q} \xi_{(k-1) Q+i} \circ f, S_{k} \circ f$ is approximately differentiable at $a$ and

$$
\text { ap } A\left(S_{k} \circ f\right)(a)=S_{k} \circ \text { ap } A f(a) .
$$

(3) If $f \in *_{2}(A, \mathbf{Q})$, then $S_{k} \circ f \in \mathrm{N}_{2}(A, \mathbf{R})$.

Proof. From

$$
\begin{aligned}
\mid \xi \circ \text { ap } A f(a) & -\operatorname{ap} A(\xi \circ f)(a) \mid \\
& \leqslant|\xi \circ \operatorname{ap} A f(a)-\xi \circ f|+|\xi \circ f-\operatorname{ap} A(\xi \circ f)(a)| \\
& \leqslant \operatorname{Lip} \xi(\operatorname{ap} A f(a), f)+|\xi \circ f-\operatorname{ap} A(\xi \circ f)(a)|
\end{aligned}
$$

it follows that $\xi \circ$ ap $A f(a)$ is approximately differentiable at $a$ and

$$
\text { ap } A(\xi \circ \text { ap } A f(a))(a)=\operatorname{ap} A(\xi \circ f)(a) .
$$

Now $\boldsymbol{\xi} \circ$ ap $A f(a)$ is piecewise affine, as it readily follows from the definition of $\boldsymbol{\xi}$, and so $\xi \circ$ ap $A f(a)$ can be approximately differentiable at $a$ only if it is affine in a neighborhood $U$ of $a$. Then

$$
\xi \circ \operatorname{ap} A f(a)|U=\operatorname{ap} A(\xi \circ \operatorname{ap} A f(a))(a)| U=\operatorname{ap} A(\xi \circ f)(a),
$$

as required in (1).

The first formula of (2) is clear by the definitions of $\boldsymbol{\xi}$ and $S_{k}$, and it implies that $S_{k} \circ f$ is approximately differentiable at $a$. Since $S_{k} \circ$ ap $A f(a)$ is affine, the second formula follows from the first and (1).

(3) is obvious by (2).

2.3. Lemma. If $A \subset \mathbf{R}^{m}$ is open and $f \in \mathrm{S}_{2}(A, \mathbf{Q})$, then

$$
\text { ( (R) } \lim _{R \rightarrow a}(\operatorname{diam} R)^{-m-2} \int_{R}\left(\mathcal{S}(f, \text { ap } A f(a))^{2} d \mathbb{L}^{m}=0\right.
$$

for $\mathfrak{L}^{\prime \prime}$ almost all $a \in A$.

Proof. By [SE, Chapter VIII, Theorem 1(b)] and Hölder's inequality,

$$
\text { ( } R \text { R ) } \lim _{R \rightarrow a}(\operatorname{diam} R)^{-m-2} \int_{R}|\xi \circ f-\operatorname{ap} A(\xi \circ f)(a)|^{2} d L^{m}=0
$$

for $\mathbb{L}^{m}$ almost all $a \in A$. The lemma follows from this, Lemma 2.2(1) and the fact that $\operatorname{Lip} \xi^{-1}<\infty$.

2.4. Lemma. Suppose $A \subset \mathbf{R}^{m}$ is bounded and open, $f, g \in \mathcal{g}_{2}(A, \mathbf{Q}), \varphi \in \mathcal{V}_{2}(A, \mathbf{R})$, $0 \leqslant \varphi \leqslant 1$, and

$$
H=H(f, g, \varphi)=\boldsymbol{\xi}^{-1} \circ \rho \circ[\xi \circ f+\varphi(\xi \circ g-\boldsymbol{\xi} \circ f)] .
$$

Then $H \in v_{2}(A, \mathbf{Q}), H(x)=f(x)$ for $x \in \varphi^{-1}\{0\}, H(x)=g(x)$ for $x \in \varphi^{-1}\{1\}$, $\mathcal{G}(H, g) \leqslant \operatorname{Lip}\left(\xi^{-1} \circ \rho\right) \operatorname{Lip} \xi \mathcal{G}(f, g)$ and for any $\mathbb{L}^{m}$ measurable set $B \subset A$

$$
\operatorname{Dir}(H ; B) \leqslant C\left[\operatorname{Dir}(f ; B)+\operatorname{Dir}(g ; B)+\int_{B}|D \varphi|^{2} \mathfrak{G}(f, g)^{2} d \mathfrak{L}^{m}\right]
$$


where $C=10(\operatorname{Lip} \rho \operatorname{Lip} \xi)^{2}$. Furthermore, if $f_{\mid \partial A}, g_{\mid \partial A} \in \partial \mathscr{Q}_{2}(\partial A, \mathbf{Q})$ and $\varphi_{\mid \partial A}=0$ (resp. $\left.\varphi_{\mid \partial A}=1\right)$, then $H_{\mid \partial A}=f_{\mid \partial A}\left(\right.$ resp. $\left.H_{\mid \partial A}=g_{\mid \partial A}\right)$.

Proof. The first four assertions are clear. We estimate $\operatorname{Dir}(H ; B)$ using the inequality $(t+s)^{2} \leqslant 2\left(t^{2}+s^{2}\right)$ to obtain

$$
\begin{aligned}
\operatorname{Dir}(H ; B) & \leqslant \operatorname{Dir}(\xi \circ H ; B) \leqslant(\operatorname{Lip} \rho)^{2} \operatorname{Dir}(\xi \circ f+\varphi(\xi \circ g-\xi \circ f) ; B) \\
& \leqslant 2(\operatorname{Lip} \rho)^{2}[\operatorname{Dir}(\xi \circ f ; B)+\operatorname{Dir}(\varphi(\xi \circ g-\xi \circ f) ; B)] \\
& \leqslant 2(\operatorname{Lip} \rho)^{2}[\operatorname{Dir}(\xi \circ f ; B)+2 \operatorname{Dir}(\xi \circ g-\xi \circ f ; B) \\
& \left.+2 \int_{B}|D \varphi|^{2}|\xi \circ g-\xi \circ f|^{2} d L^{m}\right] \\
& \leqslant 2(\operatorname{Lip} \rho \operatorname{Lip} \xi)^{2}[\operatorname{Dir}(f ; B)+4(\operatorname{Dir}(f ; B)+\operatorname{Dir}(g ; B)) \\
& \left.+2 \int_{B}|D \varphi|^{2} \mathcal{G}(f, g)^{2} d \varrho^{m}\right] \\
& \leqslant C\left[\operatorname{Dir}(f ; B)+\operatorname{Dir}(g ; B)+\int_{B}|D \varphi|^{2} \mathcal{G}(f, g)^{2} d \mathcal{L}^{m}\right] .
\end{aligned}
$$

To prove the last statement we may assume that $f, g$ and $\varphi$ are strictly defined on an open ball $U$ containing Clos $A, f, g \in \mathcal{Y}_{2}(U, \mathbf{Q})$ and $\varphi \in \mathcal{Y}_{2}(U, \mathbf{R})$. Then $\varphi(x)=0$ (resp. $\varphi(x)=1$ ) for $\mathscr{H}^{m-1}$ almost all $x \in \partial A$ and $H$ defined by the same formula belongs to $\mathcal{O}_{2}(U, \mathbf{Q})$. It is straightforward to verify that every common Lebesgue point of $\boldsymbol{\xi} \circ f, \boldsymbol{\xi} \circ g$ and $\varphi$ is also a Lebesgue point of $H$, and thus $H$ agrees $\mathfrak{H}^{m-1}$ almost everywhere on $U$ with a strictly defined function. Since $H(x)=f(x)$ (resp. $H(x)=g(x))$ for $\mathfrak{K}^{m-1}$ almost all $x \in \partial A$, it follows that $H_{\mid \partial A}=f_{\mid \partial A}$ (resp. $H_{\mid \partial A}=$ $\left.g_{\mid \partial A}\right)$.

2.5. Lemma. Suppose $A \subset \mathbf{R}^{m}$ is bounded and open, $f, g \in \mathcal{Y}_{2}(A, \mathbf{Q})$ and

$$
\varphi(x)=\mathcal{G}(f(x), g(x)) \text { for } x \in A \text {. }
$$

Then $\varphi \in \mathscr{O}_{2}(A, \mathbf{R})$ and

$$
|D \varphi(x)| \leqslant \operatorname{Lip} \xi^{-1} \operatorname{Lip} \xi(|\operatorname{ap} A f(x)|+|\operatorname{ap} A g(x)|)
$$

for $\mathcal{L}^{m}$ almost all $x \in A$. Furthermore, if $f_{\mid \partial A}=g_{\mid \partial A} \in \partial \mathcal{Y}_{2}(\partial A, \mathbf{Q})$, then $\varphi_{\mid \partial A}=0$.

Proof. We may assume $f$ and $g$ are strictly defined. For $x, y \in A$

$$
\begin{aligned}
|\varphi(x)-\varphi(y)| & \leqslant \mathcal{G}(f(x), f(y))+\mathcal{G}(g(x), g(y)) \\
& \leqslant \operatorname{Lip} \xi^{-1}(|\xi \circ f(x)-\xi \circ f(y)|+|\xi \circ g(x)-\xi \circ g(y)|) .
\end{aligned}
$$

Since $\xi \circ f$ and $\xi \circ g$ are ACL, it follows that also $\varphi$ is ACL, and for $i=1, \ldots, m$ and $\complement^{m}$ almost all $x \in A$,

$$
\left|D_{i} \varphi(x)\right| \leqslant \operatorname{Lip} \xi^{-1}\left(\left|D_{i}(\xi \circ f)(x)\right|+\left|D_{i}(\xi \circ g)(x)\right|\right) .
$$

Consequently, the partial derivatives of $\varphi$ are square summable, and $\varphi \in \mathcal{O}_{2}(A, \mathbf{R})$. The estimate for $|D \varphi(x)|$ follows immediately from the estimates for $\left|D_{i} \varphi(x)\right|$. 
We can prove the last statement in the same way as we proved the last statement of 2.4 observing that the above estimate for $|\varphi(x)-\varphi(y)|$ implies that $\varphi$ agrees $\mathfrak{H}^{m-1}$ almost everywhere with a strictly defined function.

2.6. Lemma. Suppose $f_{i}: \mathbf{R} \rightarrow \mathbf{R}, i=1,2, \ldots$, are nonnegative $L^{\prime \prime}$ measurable functions with $\sup _{i} \int f_{i} d L^{\prime}<\infty$ and that $A \subset \mathbf{R}$ with $L^{\prime}(\mathbf{R} \sim A)=0$. Then there are $a$ dense subset $D$ of $A$ and a subsequence $\left(f_{i}\right)$ of $\left(f_{i}\right)$ such that $\sup _{j} f_{i_{1}}(t)<\infty$ for all $t \in D$.

Proof. Let $I_{1}, I_{2}, \ldots$ be the dyadic intervals in $\mathbf{R}$. It follows from Fatou's lemma that $\liminf _{i} f_{i}(t)<\infty$ for $\mathcal{L}^{1}$ almost all $t \in A \cap I_{1}$; hence there are $t_{1} \in A \cap I_{1}$ and a subsequence $\left(f_{i}^{1}\right)$ of $\left(f_{i}\right)$ such that $\sup _{i} f_{i}{ }^{1}\left(t_{1}\right)<\infty$. Similarly, there are $t_{2} \in A \cap I_{2}$ and a subsequence $\left(f_{i}^{2}\right)$ of $\left(f_{i}^{1}\right)$ such that $\sup _{i} f_{i}^{2}\left(t_{2}\right)<\infty$. Continuing this process we can take $D=\left\{t_{1}, t_{2}, \ldots\right\}$ and $\left(f_{i}\right)=\left(f_{i}^{i}\right)$.

2.7. Lemma. Suppose $A \subset \mathbf{R}^{m}$ is open, $f, f_{i} \in \mathrm{N}_{2}(A, \mathbf{Q}), i=1,2, \ldots$ are strictly defined, $\lim _{i \rightarrow \infty} \int_{A} \mathcal{Q}\left(f_{i}, f\right)^{2} d L^{i m}=0$ and $\sup _{i} \operatorname{Dir}\left(f_{i} ; A\right)<\infty$. Then there are $a$ subsequence $\left(f_{i}\right)$ of $\left(f_{i}\right)$ and for each $k=1, \ldots, m$ a dense subset $D_{k}$ of $\mathbf{R}$ such that if $t \in D_{k}$ and $V=\left\{x \in \mathbf{R}^{m}: x_{k}=t\right\}$, then

$$
\lim _{j \rightarrow \infty} \int_{A \cap V} \mathcal{i}\left(f_{i}, f\right)^{2} d \mathfrak{H}^{m-1}=0 \text { and } \sup _{j} \operatorname{dir}\left(f_{i} ; A \cap V\right)<\infty \text {. }
$$

Proof. We first take $k=1$ and denote $V(t)=\left\{x \in \mathbf{R}^{m}: x_{1}=t\right\}$ for $t \in \mathbf{R}$. By Fubini's theorem the functions $t \rightarrow \int_{A \cap V(t)} \mathcal{S}\left(f_{i}, f\right)^{2} d \mathcal{H}^{m-1}$ converge to zero in the mean, and so there is a subsequence $\left(f_{i}^{0}\right)$ of $\left(f_{i}\right)$ such that

$$
\lim _{i \rightarrow \infty} \int_{A \cap V(t)}\left(f_{i}^{0}, f\right)^{2} d \mathcal{H}^{m-1}=0
$$

for $L^{\prime \prime}$ almost all $t \in \mathbf{R}$. We apply Lemma 2.6 to the functions

$$
t \mapsto \int_{A \cap V(t)}\left|\operatorname{ap} A f_{i}^{0}\right|^{2} d \mathcal{H}^{m-1}
$$

to find a dense subset $D_{1}$ of $\mathbf{R}$ and subsequence $\left(f_{i}^{1}\right)$ of $\left(f_{i}^{0}\right)$ such that for $t \in D_{1}$ (1) holds and

$$
\sup _{i} \operatorname{dir}\left(f_{i}^{1} ; A \cap V(t)\right) \leqslant \sup _{i} \int_{A \cap v^{\prime}(t)}\left|\operatorname{ap} A f_{i}^{1}\right|^{2} d \mathcal{K}^{m-1}<\infty .
$$

Next we take $k=2$ and apply the above argument to $\left(f_{i}^{1}\right)$ to obtain $D_{2}$ and a subsequence $\left(f_{i}^{2}\right)$ of $\left(f_{i}^{1}\right)$. Continuing in this manner we get $\left(f_{i}^{m}\right)$ for the required subsequence.

2.8. Lemma. Suppose $R$ and $R^{\prime}$ are open intervals in $\mathbf{R}^{m}, \operatorname{Clos} R \subset R^{\prime}$ and $f_{i} \in \mathrm{O}_{2}\left(R^{\prime}, \mathbf{R}^{k}\right), i=1,2, \ldots$, are strictly defined functions. If

$$
\lim _{i \rightarrow \infty} \int_{\partial R}\left|f_{i}\right|^{2} d \mathcal{H}^{-m-1}=0 \text { and } \sup _{i} \operatorname{dir}\left(f_{i} ; \partial R\right)<\infty
$$

then there are functions $g_{i} \in \mathrm{Q}_{2}\left(R^{\prime}, \mathbf{R}^{k}\right)$ such that $g_{i}\left|R=f_{i}\right| R, g_{\mid \partial R^{\prime}}=0$ and $\lim _{i \rightarrow \infty} \operatorname{Dir}\left(g_{i} ; R^{\prime} \sim R\right)=0$. 
Proof. Choose an open interval $R^{\prime \prime}$ with Clos $R^{\prime \prime} \subset R$ and a bi-Lipschitzian homeomorphism $F$ : Clos $R^{\prime} \sim R \rightarrow \operatorname{Clos} R \sim R^{\prime \prime}$ such that $F(x)=x$ for $x \in \partial R$ and $F\left(\partial R^{\prime}\right)=\partial R^{\prime \prime}$. The restrictions $f_{i} \mid \partial R$ are strongly in $H_{2}^{1}(\partial R)$ in the sense of [M, Definition 4.4.4] (this follows e.g. from [SE, VI, 4.3]); hence by [M, Lemma 4.4.3] and its proof there are functions $h_{i} \in \mathcal{Q}_{2}\left(R, \mathbf{R}^{k}\right)$ such that $h_{i \mid \partial R}=f_{i \mid \partial R}, h_{i \mid \partial R^{\prime \prime}}=0$ and $\lim _{i} \operatorname{Dir}\left(h_{i} ; R\right)=0$. When we define $g_{i}(x)=f_{i}(x)$ for $x \in R$ and $g_{i}(x)=h_{i} \circ F(x)$ for $x \in R^{\prime} \sim R$, then the functions $g_{i}$ have the required properties.

3. The quadratic component of a geometric integrand. Let $F: \mathcal{G}(m+n, m) \rightarrow \mathbf{R}$ be a geometric semielliptic integrand of class 3 on $\mathbf{R}^{m+n}$ with ellipticity bound $c \geqslant 0$, for the definitions see [A1, 1.2; F, 5.1.2] ( $F$ is elliptic, if $c>0$ ). The Taylor expansion of $F$ in a suitable coordinate system gives a constant $C$ and functions $L, G, H$ : $P(n, m) \rightarrow \mathbf{R}$ such that $L\left(p_{i}^{k}\right)=\Sigma l(i, k) p_{i}^{k}$, for some constants $l(i, k), G$ is a constant coefficient quadratic integrand, as defined in 1.4 , called the quadratic component of $F$ (with respect to $\mathbf{R}^{m} \times\{0\}$ ), $\lim _{p \rightarrow 0}|p|^{-2} H(p)=0$, and that

$$
\mathbf{F}\left[\left(\left[\left[\mathbf{1}_{\mathbf{R}^{m}}\right]\right] \bowtie f\right)_{\#}\left(E^{m} L U\right)\right]=C L^{m}(U)+\mathbf{L}(f ; U)+\mathbf{G}(f ; U)+\mathbf{H}(f ; U)
$$

for any bounded open set $U \subset \mathbf{R}^{m}$ and any Lipschitzian function $f: U \rightarrow \mathbf{R}^{n}$ (see $[\mathbf{A} 1,5.1]$ and, for the notation, $[\mathbf{A}, 1.6])$. This formula extends to Lipschitzian functions $f: U \rightarrow \mathbf{Q}$ by $[\mathbf{A}, 1.5(10)(i v)]$ when $C \mathscr{L}(U)$ is replaced by $C Q^{\complement m}(U)$.

Suppose $f: \mathbf{R}^{m} \rightarrow \mathbf{Q}$ is a Lipschitzian function with compact support. Then Lemma 2.2(2) implies

$$
\begin{aligned}
\mathbf{L}\left(f ; \mathbf{R}^{m}\right) & =\sum_{i, k} l(i, k) \int D_{i}\left(S_{k} \circ \text { ap } A f(x)\right) d \varrho^{m} x \\
& =\sum_{i, k} l(i, k) \int D_{i}\left(S_{k} \circ f\right) d L^{m}=0,
\end{aligned}
$$

because $S_{k} \circ f$ has compact support. The argument of $[\mathbf{A} 1,5.4(2)]$ now applies to prove

3.1. LeMMA. The quadratic component of a geometric (semi) elliptic integrand, is $Q$ ( semi ) elliptic.

\section{Lower semicontinuity of a $Q$ semielliptic integral.}

Throughout this section we assume that $G$ is $Q$ semielliptic and that $R \subset \mathbf{R}^{m}$ is an open interval.

4.1. Lemma. If $f \in \mathcal{O}_{2}(R, \mathbf{Q}), h \in A(n, m)$ and $f_{\mid \partial R}=Q[[h]] \mid \partial R$, then $\mathbf{G}(f, R) \geqslant$ $Q \mathbf{G}(h, R)$.

Proof. We assume, as we may, that $f$ is strictly defined. We set according to $[\mathbf{A}, 2.3(2)] g=f \oplus(-h)$, that is,

$$
g(x)=\sum_{\nu=1}^{Q}\left[\left[y^{\nu}-h(x)\right]\right] \quad \text { when } f(x)=\sum_{\nu=1}^{Q}\left[\left[y^{\nu}\right]\right] .
$$


Then $g \in \mathrm{g}_{2}(R, \mathbf{Q})$ and $g_{\mid \partial R}=0$. Suppose $x \in A, \boldsymbol{\xi} \circ f$ is approximately differentiable at $x$ and $\operatorname{ap} A f(x)=\sum_{\nu=1}^{Q}\left[\left[h^{\nu}\right]\right]$. Then one easily checks that ap $A g(x)=$ $\sum_{\nu=1}^{Q}\left[\left[h^{\nu}-h\right]\right]$. Consequently, since $q(i, j, k, l)=q(j, i, l, k)$,

$$
\begin{aligned}
G g(x) & =\sum_{i, j, k, l, \nu} q(i, j, k, l)\left(D_{i} h_{k}^{\nu}-D_{i} h_{k}\right)\left(D_{j} h_{l}^{\nu}-D_{j} h_{l}\right) \\
& =G f(x)+Q G h(x)-2 \sum_{i, j, k, l, \nu} q(i, j, k, l) D_{i} h_{k}^{\nu} D_{j} h_{l} \\
& =G f(x)+Q G h(x)-2 \sum_{i, j, k, l} q(i, j, k, l) D_{j} h_{l} \sum_{\nu=1}^{Q} D_{i} h_{k}^{\nu} \\
& =G f(x)+Q G h(x)-2 \sum_{i, j, k, l} q(i, j, k, l) D_{j} h_{l} D_{i}\left(S_{k} \circ \text { ap } A f(x)\right) \\
& =G f(x)+Q G h(x)-2 \sum_{i, j, k, l} q(i, j, k, l) D_{j} h_{l} D_{i}\left(S_{k} \circ f\right)(x)
\end{aligned}
$$

according to 2.2(2). Since $S_{k} \circ f$ is $\mathrm{ACL}$, as $\xi \circ f$ is $\mathrm{ACL}$, and $S_{k} \circ f_{\mid \partial R}=Q h_{k} \mid \partial R$, we have by Fubini's theorem

$$
\int_{R} D_{i}\left(S_{k} \circ f\right) d L^{m}=Q \int_{R} D_{i} h_{k} d L^{m}
$$

Therefore by the $Q$ semiellipticity of $G$

$$
\begin{aligned}
0 \leqslant & \mathbf{G}(g ; R)=\mathbf{G}(f ; R)+Q \mathbf{G}(h ; R) \\
& -2 \sum_{i, j, k, l} q(i, j, k, l) D_{j} h_{l} \int_{R} D_{i}\left(S_{k} \circ f\right) d L^{i m} \\
= & \mathbf{G}(f ; R)-Q \mathbf{G}(h ; R),
\end{aligned}
$$

which proves the lemma.

4.2. Lemma. Suppose $f \in \mathcal{Q}_{2}(R, \mathbf{Q}), h=\sum_{\nu=1}^{Q} h^{\nu}: \mathbf{R}^{m} \rightarrow \mathbf{Q}$ is affine and

$$
0<\lambda \leqslant \inf \left\{\left|h^{\mu}(x)-h^{\nu}(x)\right|: x \in R, h^{\mu} \neq h^{\nu}\right\}
$$

(with the agreement inf $\phi=\infty)$. If $f_{\mid \partial R}=h_{\mid \partial R}$ and $\mathcal{S}(f(x), h(x))<\lambda / 4$ for $L^{m}$ almost all $x \in R$, then $\mathbf{G}(f ; R) \geqslant \mathbf{G}(h ; R)$.

Proof. We may assume $f$ is strictly defined. There are $J \subset\{1, \ldots, Q\}$ and positive integers $Q_{i}, i \in J$, such that $\Sigma_{i \in J} Q_{i}=Q, h^{i} \neq h^{j}$ for $i \neq j, i, j \in J$, and $h=$ $\sum_{i \in J} Q_{i}\left[\left[h^{i}\right]\right]$. We can write $f$ as $f=\sum_{i \in J} f_{i}$ by requiring that $f_{i}: R \rightarrow \mathbf{Q}_{i}$ and $\mathcal{G}\left(f_{i}(x), Q_{i}\left[\left[h^{i}(x)\right]\right]\right)<\lambda / 4$ whenever $\mathcal{S}(f(x), h(x))<\lambda / 4$. It follows from the definition of $\mathcal{G}$ and the assumptions on $f$ and $h$ that if $x, y \in R, \mathcal{G}(f(x), h(x))<\lambda / 4$, $\mathcal{G}(f(y), h(y))<\lambda / 4$ and $x$ and $y$ are sufficiently close to each other, then

$$
\mathcal{G}\left(f_{i}(x), f_{i}(y)\right) \leqslant \mathcal{G}(f(x), f(y)) \text { for } i \in J \text {. }
$$

From this and the ACL characterization of the $\mathscr{\mathcal { H }}_{2}$ spaces, it follows that $f_{i} \in$ $\stackrel{Q}{~}_{2}\left(R, \mathbf{Q}_{i}\right)$ for $i \in J$. Obviously $f_{i \mid \partial R}=Q_{i}\left[\left[h^{i}\right]\right] \mid \partial R$ and ap $A f(x)=\sum_{i \in J}$ ap $A f_{i}(x)$ for $L^{m}$ almost all $x \in R$. Hence Lemma 4.1 gives

$$
G(h ; R)=\sum_{i \in J} Q_{i} \mathbf{G}\left(h_{i} ; R\right) \leqslant \sum_{i \in J} \mathbf{G}\left(f_{i} ; R\right)=\mathbf{G}(f ; R) .
$$


4.3. LEMMA. For any $g \in \partial \mathcal{Y}_{2}(\partial R, \mathbf{Q})$,

$$
\inf \left\{\mathbf{G}(f ; R): f \in \mathcal{Y}_{2}(R, \mathbf{Q}), f_{\mid \partial R}=g\right\}>-\infty .
$$

Proof. There is $h \in \mathcal{Y}_{2}(2 R, \mathbf{Q})$ such that $h_{\mid \partial R}=g$ and $h_{\mid \partial 2 R}=0$. If $f \in \mathcal{Y}_{2}(R, \mathbf{Q})$ and $f_{\mid \partial R}=g$, we can extend $f$ to $2 R$ as $f|2 R \sim R=h| 2 R \sim R$. By the semiellipticity of $G, \mathbf{G}(f ; 2 R) \geqslant 0$, whence

$$
\mathbf{G}(f ; R)=\mathbf{G}(f ; 2 R)-\mathbf{G}(h ; 2 R \sim R) \geqslant-\mathbf{G}(h ; 2 R \sim R),
$$

which proves the lemma.

4.4. Lemma If $f \in \mathcal{Y}_{2}(R, \mathbf{Q}), c \in \mathbf{Q}$ and $f_{\mid \partial R}=c$, then $G(f ; R) \geqslant 0$.

Proof. Suppose $\mathbf{G}(f, R)=\alpha<0$. We may assume that $R$ is a cube centered at the origin, since we can always extend $f$ outside $R$ as the constant $c$. Let $d$ be the side-length of $R$. For each positive integer $k$ we divide $R$ into $k^{m}$ cubes $R_{1}, \ldots, R_{k^{m}}$ with the side-length $d / k$ and centres $x_{1}, \ldots, x_{k^{m}}$. We define

$$
g_{k}(x)=f\left(k\left(x-x_{i}\right)\right) \text { for } x \in R_{i}, i=1, \ldots, k^{m} .
$$

Since $g_{k \mid \partial R_{1}}=c$ for each $i, g_{k} \in \mathscr{Y}_{2}(R, \mathbf{Q})$ and $g_{k \mid \partial R}=c$. As

$$
G g_{k}(x)=k^{2} G f\left(k\left(x-x_{i}\right)\right) \text { for } \mathcal{L}^{m} \text { almost all } x \in R_{i} \text {, }
$$

we have

$$
\begin{aligned}
\mathbf{G}\left(g_{k} ; R_{i}\right) & =k^{2} \int_{R_{i}} G f\left(k\left(x-x_{i}\right)\right) d \varrho^{m} x \\
& =k^{2-m} \int_{R} G f(x) d \varrho^{m} x=k^{2-m} \alpha,
\end{aligned}
$$

and $\mathbf{G}\left(g_{k} ; R\right)=\sum_{i=1}^{k^{m}} \mathbf{G}\left(g_{k} ; R_{i}\right)=k^{2} \alpha \rightarrow-\infty$ as $k \rightarrow \infty$. This contradicts Lemma 4.3.

A more precise form of the above lemma will be given in 6.2 .

4.5. Lemma. There is a constant $C$ depending only on $m, n, Q$ and $G$ such that if $R \in \mathcal{R}, f, g \in \mathcal{Y}_{2}(R, \mathbf{Q})$ and $f_{\mid \partial R}=g_{\mid \partial R} \in \partial \mathcal{Y}_{2}(\partial R, \mathbf{Q})$ then $\mathbf{G}(g ; R) \geqslant-C \operatorname{Dir}(f ; R)$.

Proof. We may use a linear transformation of $\mathbf{R}^{m}$ to reduce the proof to the case where $R$ is a unit cube in $\mathbf{R}^{m}$. We denote by \|\|$_{2}$ the usual $L^{2}$-norm and we use the norm \|\|$_{2,2}=\|\|_{2}+\operatorname{Dir}^{1 / 2}$ on $\mathcal{Y}_{2}(R, Q)$. Below $C_{1}, C_{2}$ and $C_{3}$ will be positive constants depending only on $m, n, Q$ and $G$.

By Poincare inequality [GT, p. 157, (7.45)] there is a constant $\lambda \in \mathbf{R}^{P Q}$ such that

$$
\|\xi \circ f-\lambda\|_{2} \leqslant C_{1}(\operatorname{Dir}(\xi \circ f ; R))^{1 / 2} \text {. }
$$

Using [M, 3.4.3] we find $\varphi \in \mathcal{O}_{2}\left(2 R, \mathbf{R}^{P Q}\right)$ such that $\varphi_{\mid \partial 2 R}=0, \varphi \mid R=\boldsymbol{\xi} \circ f-\lambda$, $\varphi_{\mid \partial R}=\xi \circ f-\lambda_{\mid \partial R}$ and $\|\varphi\|_{2,2} \leqslant C_{2}\|\xi \circ f-\lambda\|_{2,2}$. We set $h=\xi^{-1} \circ \rho \circ(\varphi+\lambda)$. Then $h \in \mathcal{O}_{2}(2 R, \mathbf{Q}), h_{\mid \partial R}=f_{\mid \partial R}=g_{\mid \partial R}, h_{\mid \partial 2 R}=\xi^{-1} \circ \rho(\lambda)$, and

$$
\begin{aligned}
\operatorname{Dir}(h ; 2 R)^{1 / 2} & \leqslant \operatorname{Dir}(\xi \circ h ; 2 R)^{1 / 2} \\
& \leqslant \operatorname{Lip} \rho \operatorname{Dir}(\varphi ; 2 R)^{1 / 2} \leqslant C_{2} \operatorname{Lip} \rho\|\xi \circ f-\lambda\|_{2,2} \\
& \leqslant C_{2} \operatorname{Lip} \rho\left(C_{1}+1\right) \operatorname{Dir}(\xi \circ f, R)^{1 / 2} \leqslant C_{3} \operatorname{Dir}(f ; R)^{1 / 2}
\end{aligned}
$$


We define $\tilde{g} \in \mathscr{\vartheta}_{2}(2 R, \mathbf{Q})$ by $\tilde{g}|R=g| R$ and $\tilde{g}|2 R \sim R=h| 2 R \sim R$. Then $\tilde{g}_{\mid 22 R}=$ $h_{\mid \partial 2 R}$ is constant. Therefore Lemma 4.4 implies $\mathbf{G}(\tilde{g} ; 2 R) \geqslant 0$, and thus

$\mathbf{G}(g ; R)=\mathbf{G}(\tilde{g} ; 2 R)-\mathbf{G}(h ; 2 R \sim R) \geqslant-C_{0} \operatorname{Dir}(h ; 2 R \sim R) \geqslant-C_{0} C_{3}^{2} \operatorname{Dir}(f ; R)$, and we may take $C=C_{0} C_{3}^{2}$.

4.6. Lemma. There is a constant $C$ depending only on $m, n, Q$ and $G$, and, given positive numbers $M, \varepsilon$ and $\lambda$, there are positive numbers $\eta$ and $\delta$ with the following property:

If $R$ is any open interval in $\mathbf{R}^{m}, d=\operatorname{diam} R, h=\sum_{\nu=1}^{Q}\left[\left[h^{\nu}\right]\right]: \mathbf{R}^{m} \rightarrow \mathbf{Q}$ is affine,

$$
\inf \left\{\left|h^{\mu}(x)-h^{\nu}(x)\right|: x \in R, h^{\mu} \neq h^{\nu}\right\} \geqslant \lambda \geqslant d / \eta
$$

and $\operatorname{Lip} h \leqslant M$, then

$$
\begin{aligned}
\mathbf{G}(f ; R) \geqslant \mathbf{G}(h ; R)-C(\varepsilon \operatorname{Dir}(f ; R)+ & \varepsilon M^{2} L^{2 m}(R) \\
& +M^{2} L^{m}\{x \in R:(\mathcal{S}(f(x), h(x))>\delta\})
\end{aligned}
$$

whenever $f \in \mathrm{P}_{2}(R, \mathbf{Q})$ with $f_{\mid \partial R}=h \mid \partial R$.

Proof. We set

$$
\begin{aligned}
& C_{1}=10(\operatorname{Lip} \rho \operatorname{Lip} \xi)^{2}, \quad C_{2}=C_{1}\left(1+18\left(\operatorname{Lip} \xi^{-1} \operatorname{Lip} \xi\right)^{2}\right), \\
& C=C_{0}\left(2 C_{2}+1\right), \quad \eta=\left(32 M \operatorname{Lip}\left(\xi^{-1} \circ \rho\right) \operatorname{Lip} \xi\right)^{-1} 2^{-2 / \varepsilon},
\end{aligned}
$$

choose integers $k_{0}$ and $k_{1}$ such that

$$
2^{-k_{0}-1}<\left(16 \operatorname{Lip}\left(\xi^{-1} \circ \rho\right) \operatorname{Lip} \xi\right)^{-1} \lambda \leqslant 2^{-k_{0}}, \quad k_{1}-1 \leqslant k_{0}+2 / \varepsilon<k_{1},
$$

and take $\delta=2^{-k_{1}}$.

Let $h, R$ and $d$ be as above, and let $f \in \mathrm{S}_{2}(R, \mathbf{Q})$ with $f_{\mid \partial R}=h \mid \partial R$. We note that $d$ Lip $h \leqslant \eta \lambda M \leqslant \delta$. The sets

$$
A_{k}=\left\{x \in R: 2^{-k} \leqslant \mathcal{S}(f(x), h(x))<2^{-k+1}\right\}, \quad k=k_{0}, k_{0}+1, \ldots, k_{1},
$$

are disjoint and $\mathrm{L}^{m}$ measurable and, since $k_{1}-k_{0}>2 / \varepsilon$, there is $k$ such that setting $A=A_{k}$, we have

$$
\operatorname{Dir}(f ; A) \leqslant \varepsilon \operatorname{Dir}(f ; R), \quad \operatorname{Dir}(h ; A) \leqslant \varepsilon \operatorname{Dir}(h ; R) .
$$

We also denote $\zeta=2^{-k}$ and

$$
B=\{x \in R: \mathcal{G}(f(x), h(x))<\zeta\}, \quad D=\{x \in R: \mathcal{G}(f(x), h(x)) \geqslant 2 \zeta\} .
$$

We define $\psi: \mathbf{R} \rightarrow \mathbf{R}$ setting $\psi(t)=0$ for $t \leqslant \zeta, \psi(t)=(t-\zeta) / \zeta$ for $\zeta \leqslant t \leqslant 2 \zeta$, and $\psi(t)=1$ for $t \geqslant 2 \zeta$, and $\varphi: R \rightarrow \mathbf{Q}$ by

$$
\varphi(x)=\psi(\mathcal{G}(f(x), h(x))) \text { for } x \in R .
$$

Then by Lemma 2.5, $\varphi \in \mathcal{O}_{2}(R, \mathbf{R}), \varphi_{\mid \partial R}=0$ and

$$
|D \varphi(x)| \leqslant \zeta^{-1} \operatorname{Lip} \xi^{-1} \operatorname{Lip} \xi(|\operatorname{ap} A f(x)|+|h|)
$$

for $L^{m}$ almost all $x \in R$. Fix $a \in R$. Then

$$
\mathcal{G}(h(x), h(a)) \leqslant d \operatorname{Lip} h \leqslant \delta \leqslant \zeta \quad \text { for } x \in R .
$$


Recalling Lemma 2.4 we define $g=H(h(a), f, \varphi) \in \mathcal{Y}_{2}(R, \mathbf{Q})$ and have $g \mid B=h(a)$, $g|D=f| D, g_{\mid \partial R}=h(a)$, and, since $\mathcal{G}(f(x), h(a)) \leqslant 3 \zeta$ for $x \in A$,

$$
\begin{aligned}
\operatorname{Dir}(g ; A) & \leqslant C_{1}\left(\operatorname{Dir}(f ; A)+\int_{A}|D \varphi|^{2} \mathcal{G}(f, h(a))^{2} d \mathcal{L}^{m}\right) \\
& \leqslant C_{1}\left(\operatorname{Dir}(f ; A)+9\left(\operatorname{Lip} \xi^{-1} \operatorname{Lip} \xi\right)^{2} \int_{A}(|\operatorname{ap} A f(x)|+|h|)^{2} d \mathcal{L}^{{ }^{m} x}\right) \\
& \leqslant C_{2}(\operatorname{Dir}(f ; A)+\operatorname{Dir}(h ; A)) \\
& \leqslant C_{2} \varepsilon(\operatorname{Dir}(f ; R)+\operatorname{Dir}(h ; R)) .
\end{aligned}
$$

Since $g_{\mid \partial R}$ is constant, Lemma 4.4 implies $\mathbf{G}(g, R) \geqslant 0$. Therefore, as $g$ is constant on $B$ and $g=f$ on $D$,

$$
\begin{aligned}
0 & \leqslant \mathbf{G}(g ; R)=\mathbf{G}(g ; A)+\mathbf{G}(f ; D) \\
& \leqslant C_{0} \operatorname{Dir}(g ; A)+\mathbf{G}(f ; D) \\
& \leqslant C_{0} C_{2} \varepsilon(\operatorname{Dir}(f ; R)+\operatorname{Dir}(h ; R))+\mathbf{G}(f ; D),
\end{aligned}
$$

and

$$
\mathbf{G}(f ; D) \geqslant-C_{0} C_{2} \varepsilon(\operatorname{Dir}(f ; R)+\operatorname{Dir}(h ; R)) .
$$

Next we define, according to Lemma $2.4, \tilde{g}=H(f, h, \varphi) \in \mathcal{Y}_{2}(R, \mathbf{Q})$, where $\varphi$ is as above. Then $\tilde{g}|B=f| B, \tilde{g}|D=h| D,\left.\tilde{g}\right|_{\partial R}=f_{\mid \partial R}=h \mid \partial R$,

$$
\mathcal{S}(\tilde{g}(x), h(x)) \leqslant \operatorname{Lip}\left(\xi^{-1} \circ \rho\right) \operatorname{Lip} \xi \cdot 2 \zeta<\lambda / 4 \text { for } x \in R,
$$

and, as before,

$$
\begin{aligned}
\operatorname{Dir}(\tilde{g} ; A) & \leqslant C_{1}\left(\operatorname{Dir}(f ; A)+\operatorname{Dir}(h ; A)+\int_{A}|D \varphi|^{2} \mathcal{G}(f, h)^{2} d \mathcal{L}^{m}\right) \\
& \leqslant C_{2} \varepsilon(\operatorname{Dir}(f ; R)+\operatorname{Dir}(h ; R)) .
\end{aligned}
$$

Lemma 4.2 yields $\mathbf{G}(\tilde{g} ; R) \geqslant \mathbf{G}(h ; R)$; consequently

$$
\begin{aligned}
\mathbf{G}(f ; R)= & G(\tilde{g} ; B)+\mathbf{G}(f ; A)+\mathbf{G}(f ; D) \\
= & \mathbf{G}(\tilde{g} ; R)-\mathbf{G}(\tilde{g} ; A)-\mathbf{G}(h ; D)+G(f ; A)+G(f ; D) \\
\geqslant & \mathbf{G}(h ; R)-C_{0}(\operatorname{Dir}(\tilde{g} ; A)+\operatorname{Dir}(h ; D)+\operatorname{Dir}(f ; A) \\
& \left.\quad+C_{2} \varepsilon(\operatorname{Dir}(f ; R)+\operatorname{Dir}(h ; R))\right) \\
\geqslant & \mathbf{G}(h ; R)-C_{0}\left(\left(2 C_{2}+1\right) \varepsilon \operatorname{Dir}(f ; R)+2 C_{2} \varepsilon M^{2} \varrho^{m}(R)+M^{2} \varrho^{m}(D)\right) \\
\geqslant & \mathbf{G}(h ; R)-C\left(\varepsilon \operatorname{Dir}(f, R)+\varepsilon M^{2} \varrho^{m}(R)\right. \\
& \left.\quad+M^{2} \varrho^{m}\{x \in R: \mathcal{G}(f(x), h(x))>\delta\}\right) .
\end{aligned}
$$

4.7. TheOREM. Suppose $R \subset \mathbf{R}^{m}$ is an open interval $f, f_{i} \in \mathcal{Y}_{2}(R, \mathbf{Q}), g, g_{i} \in$ $\partial \mathcal{Q}_{2}(\partial R, \mathbf{Q}), f_{\mid \partial R}=g, f_{i \mid \partial R}=g_{i}$ for $i=1,2, \ldots$,

$$
\begin{aligned}
& \lim _{i \rightarrow \infty} \int_{R} \mathcal{G}\left(f_{i}, f\right)^{2} d \mathcal{L}^{m}=\lim _{i \rightarrow \infty} \int_{\partial R} \mathcal{G}\left(g_{i}, g\right)^{2} d \mathcal{H}^{m-1}=0, \\
& \quad \sup \operatorname{Dir}\left(f_{i} ; R\right)<\infty \text { and } \sup \operatorname{dir}\left(g_{i} ; \partial R\right)<\infty .
\end{aligned}
$$

Then $\mathbf{G}(f ; R) \leqslant \liminf _{i \rightarrow \infty} \mathbf{G}\left(f_{i} ; R\right)$. 
Proof. We may assume that the functions $f$ and $f_{i}$ are strictly defined, that the numbers $\operatorname{Dir}(f ; R), \operatorname{Dir}\left(f_{i} ; R\right)$ and $E^{m}(R)$ are all at most one and that the limit $\lim _{i} \mathbf{G}\left(f_{i}, R\right)$ exists. We extend $f$ and $f_{i}$ to clos $R$ letting $f \mid \partial R=g$ and $f_{i} \mid \partial R=g_{i}$.

Let $0<\zeta<1 / 3$. There is $\sigma, 0<\sigma<\zeta$, such that for any $\mathbb{L}^{m}$ measurable set $A \subset R$

$$
\mathscr{L}^{m}(A)<\sigma \text { implies } \operatorname{Dir}(f ; A)<\zeta \text { and }|\mathbf{G}(f ; A)|<\zeta .
$$

If $h=\sum_{\nu=1}^{Q}\left[\left[h^{\nu}\right]\right]: \mathbf{R}^{m} \rightarrow \mathbf{Q}$ is affine we set for $x \in \mathbf{R}^{m}$

$$
\lambda_{h}(x)=\inf \left\{\left|h^{\mu}(x)-h^{\nu}(x)\right|: h^{\mu} \neq h^{\nu}\right\} .
$$

Since $f$ is strongly approximately affinely approximable at $L^{m}$ almost all points of $R$, $\lambda_{\text {ap } A f(x)}(x)>0$ for $\left[^{m}\right.$ almost all $x \in R$. Hence there are positive numbers $\lambda$ and $M, M>1$, and a Borel set $A \subset R$ such that $\mathbb{L}^{m}(R \sim A)<\sigma$,

$$
\lambda_{\text {ap } A f(x)}(x)>\lambda \text { and } \operatorname{Lip} \operatorname{ap} A f(x) \leqslant|\operatorname{ap} A f(x)|<M \quad \text { for } x \in A .
$$

Set $\tau=\left(1+\sigma /\left(2 M^{2}\right)\right)^{1 / 2 m}-1$. We have by 1.5 and Lemma 2.3 for $\stackrel{L}{ }^{m}$ almost all $x \in A$

$$
\text { (으. } \lim _{S \rightarrow x^{2}} \mathbb{L}^{m}(S)^{-1} \mathbf{G}(f ; S)=G f(x)=L^{2 m}(S)^{-1} \mathbf{G}(\operatorname{ap} A f(x) ; S)
$$

for any $S \in \mathbb{R}$, as $G$ ap $A f(x)(y)=G f(x)$ for all $y \in \mathbf{R}^{m}$,

$$
\left(\text { ( R ) } \lim _{S \rightarrow x} L^{m}\left((1+\tau)^{2} S \sim S\right)^{-1} \operatorname{Dir}\left(f ;(1+\tau)^{2} S \sim S\right)=|\operatorname{ap} A f(x)|^{2}\right.
$$

and

$$
\text { ( } R) \lim _{S \rightarrow x}(\operatorname{diam} S)^{-m-2} \int_{S} \mathcal{S}(f, \text { ap } A f(x))^{2} d L^{m}=0 .
$$

Let $\varepsilon=\tau^{2} \zeta / M^{2}$ and let $\eta$ and $\delta$ be the positive numbers given by Lemma 4.6 corresponding to $M, \varepsilon$ and $\lambda$. By Vitali's covering theorem we can find disjoint intervals $R_{k} \in \mathcal{R}, k=1, \ldots, p$, with centres $x_{k} \in A$ and diameters $d_{k}$ such that $(1+\tau)^{2} R_{k} \subset R$ and

$$
\begin{aligned}
& d_{k}<\eta \lambda, \\
& \lambda_{h_{h}}(x)>\lambda \text { for } x \in R_{k} \text { where } h_{k}=\text { ap } A f\left(x_{k}\right) \text {, } \\
& \mathrm{E}^{m}\left(R \sim \bigcup_{k=1}^{p} R_{k}\right)<\sigma, \\
& \mathbf{G}\left(f ; R_{k}\right)<\mathbf{G}\left(h_{k} ; R_{k}\right)+\zeta^{2 m}\left(R_{k}\right) \text {, } \\
& \operatorname{Dir}\left(f ;(1+\tau)^{2} R_{k} \sim R_{k}\right)<2 M^{2} \mathbb{L}^{m}\left((1+\tau)^{2} R_{k} \sim R_{k}\right)<\zeta E^{m}\left(R_{k}\right) \text {. } \\
& \int_{R_{h}} \mathcal{G}\left(f, h_{k}\right)^{2} d \mathfrak{L}^{m}<\varepsilon d_{k}^{m+2}, \\
& \mathfrak{Q}^{m}\left\{x \in R_{k}: \mathcal{G}\left(f(x), h_{k}(x)\right)>\delta / 2\right\}<\varepsilon^{\mathfrak{E}^{m}}\left(R_{k}\right) .
\end{aligned}
$$

Here (8) follows from the definition of ap $A f\left(x_{k}\right)$. Moreover, passing to a subsequence without changing the notation, we may select $R_{k}$ 's so that the faces of $\partial R_{k}$ 
are contained in hyperplanes $V$ given by Lemma 2.7. Hence for $k=1, \ldots, p$

$$
\lim _{i \rightarrow x} \int_{\partial R_{h}} \mathcal{S}\left(f_{i}, f\right)^{2} d \mathcal{K}^{m-1}=0
$$

and

$$
\sup \operatorname{dir}\left(f_{i} ; \partial R_{k}\right)<\infty
$$

From (1), (4) and (5) we get

$$
\mathbf{G}(f ; R) \leqslant \sum_{k=1}^{p} \mathbf{G}\left(h_{k} ; R_{k}\right)+2 \zeta .
$$

By partitioning $R \sim \cup_{k=1}^{p} R_{k}$ into suitable intervals, we find intervals $R_{p+1}, \ldots, R_{q} \in$ Q $R$ such that $\operatorname{Clos} R=\cup_{k=1}^{q}$ Clos $R_{k}$, all the intervals $R_{k}, k=$ $1, \ldots, q$, are pairwise disjoint and that (9) and (10) hold for all $k=1, \ldots, q$. We denote $R_{k}^{\prime}=(1+\tau)^{2} R_{k}$ and choose intervals $R_{k}^{\prime \prime} \in G R$ such that Clos $R_{k} \subset R_{k}^{\prime \prime} \subset$ $(1+\tau) R_{k}$ and

$$
\operatorname{Dir}\left(f ; R_{k}^{\prime \prime} \sim R_{k}\right)<\zeta \zeta^{m}\left(R_{k}\right) .
$$

In the following $C_{1}, C_{2}, \ldots$ will denote positive constants depending only on $m, n, Q$ and $G$. Let $k=1, \ldots, q$. By (9), (10) and Lemma 2.8 there are functions $\varphi_{k, i} \in \mathcal{O}_{2}\left(R_{k}^{\prime \prime}, \mathbf{R}^{P Q}\right)$ such that $\varphi_{k, i}\left|R_{k}=\xi \circ f_{i}-\xi \circ f\right| R_{k}, \varphi_{k, \mid \gamma R_{k}^{\prime \prime}}=0$ and $\lim _{i \rightarrow \infty} \operatorname{Dir}\left(\varphi_{k, i} ; R_{k}^{\prime \prime} \sim R_{k}\right)=0$. In the following we take $i$ so large that

$$
\operatorname{Dir}\left(\varphi_{k, i}, R_{k}^{\prime \prime} \sim R_{k}\right)<\zeta \complement^{m}\left(R_{k}\right) \text {. }
$$

Set

$$
g_{k, i}=\xi^{-1} \circ \rho \circ\left(\xi \circ f \mid R_{k}^{\prime \prime}+\varphi_{k, i}\right) \in \mathcal{Y}_{2}\left(R_{k}^{\prime \prime} ; \mathbf{Q}\right) .
$$

Then $g_{k, i}\left|R_{k}=f_{i}\right| R_{k}, g_{k, \mid \partial R_{h}^{\prime \prime}}=f_{\mid \partial R_{h}^{\prime \prime}}$ and

$$
\begin{aligned}
\operatorname{Dir}\left(g_{k, i} ; R_{k}^{\prime \prime} \sim R_{k}\right) & \leqslant(\operatorname{Lip} \rho)^{2} \operatorname{Dir}\left(\xi \circ f+\varphi_{k, i} ; R_{k}^{\prime \prime} \sim R_{k}\right) \\
& \leqslant 2(\operatorname{Lip} \rho)^{2}\left[(\operatorname{Lip} \xi)^{2} \operatorname{Dir}\left(f ; R_{k}^{\prime \prime} \sim R_{k}\right)+\operatorname{Dir}\left(\varphi_{k, i} ; R_{k}^{\prime \prime} \sim R_{k}\right)\right] \\
& \leqslant C_{1} \zeta \varrho^{m}\left(R_{k}\right)
\end{aligned}
$$

by (12). Therefore

$$
\begin{aligned}
\mathbf{G}\left(f_{i} ; R_{k}\right) & =\mathbf{G}\left(g_{k, i} ; R_{k}^{\prime \prime}\right)-\mathbf{G}\left(g_{k, i} ; R_{k}^{\prime \prime} \sim R_{k}\right) \\
& \geqslant \mathbf{G}\left(g_{k, i} ; R_{k}^{\prime \prime}\right)-C_{0} \operatorname{Dir}\left(g_{k, i} ; R_{k}^{\prime \prime} \sim R_{k}\right) \\
& \geqslant G\left(g_{k, i} ; R_{k}^{\prime \prime}\right)-C_{2} \zeta^{m}\left(R_{k}\right) .
\end{aligned}
$$

Since $g_{k, \mid \partial R_{k}^{\prime \prime}}=f_{\mid \partial R_{h}^{\prime \prime}}$, Lemma 4.5 yields a constant $C_{3}$ such that, by (14) and (12),

$$
\begin{aligned}
\mathbf{G}\left(f_{i} ; R_{k}\right) & \geqslant \mathbf{G}\left(g_{k, i} ; R_{k}^{\prime \prime}\right)-C_{2} \zeta \varrho^{m}\left(R_{k}\right) \\
& \geqslant-C_{3} \operatorname{Dir}\left(f ; R_{k}^{\prime \prime}\right)-C_{2} \zeta \varrho^{m}\left(R_{k}\right) \geqslant-C_{3} \operatorname{Dir}\left(f ; R_{k}\right)-C_{4} \zeta \varrho^{m}\left(R_{k}\right) .
\end{aligned}
$$


Let $k=1, \ldots, p$. We choose a function $\varphi_{k}: \mathbf{R}^{m} \rightarrow \mathbf{R}$ of class 1 such that $0 \leqslant \varphi_{h} \leqslant 1$, $\varphi_{h}(x)=0$ for $x \in R_{k}^{\prime \prime}, \varphi_{k}(x)=1$ for $x \in \mathbf{R}^{m} \sim R_{k}^{\prime}$ and $\left|D \varphi_{k}(x)\right| \leqslant C_{5} /\left(\tau d_{h}\right)$ for $x \in \mathbf{R}^{m}$. This is possible because $(1+\tau) R_{k}^{\prime \prime} \subset R_{k}^{\prime}$. We use Lemma 2.4 to define $g_{k}=H\left(f, h_{k}, \varphi_{k}\right) \in \vartheta_{2}\left(R_{k}^{\prime}, \mathbf{Q}\right)$ for which $g_{k \mid \partial R_{h}^{\prime \prime}}=f_{\mid \partial R_{h}^{\prime \prime}}, g_{k \mid \partial R_{h}^{\prime}}=h_{k \mid \partial R_{k}^{\prime}}$ and, recalling (6) and (7),

$\operatorname{Dir}\left(g_{k} ; R_{k}^{\prime} \sim R_{k}^{\prime \prime}\right)$

$$
\begin{aligned}
& \leqslant C_{6}\left[\operatorname{Dir}\left(f ; R_{h}^{\prime} \sim R_{h}^{\prime \prime}\right)+\operatorname{Dir}\left(h_{h} ; R_{k}^{\prime} \sim R_{h}^{\prime \prime}\right)+\int_{R_{h}^{\prime} \sim R_{h}^{\prime \prime}}\left|D \varphi_{k}\right| \Theta\left(f, h_{h}\right)^{2} d L^{\prime m}\right] \\
& \leqslant C_{6}\left[\zeta^{\prime m}\left(R_{k}\right)+M^{2} \mathscr{L}^{\prime m}\left(R_{h}^{\prime} \sim R_{h}^{\prime \prime}\right)+C_{5}^{2} \tau^{-2} \varepsilon d_{k}^{m}\right] \\
& \leqslant C_{7} \zeta \check{L}^{\prime m}\left(R_{h}\right),
\end{aligned}
$$

because $L^{\prime \prime \prime}\left(R_{k}^{\prime} \sim R_{k}^{\prime \prime}\right)<\zeta / M^{2}$ and $\varepsilon<\tau^{2} \zeta$. We extend $g_{k, i}$ to $R_{k}^{\prime}$ letting $g_{k, i}(x)=$ $g_{k}(x)$ for $x \in R_{k}^{\prime} \sim R_{k}^{\prime \prime}$. Then $g_{k, i} \in V_{2}^{\prime}\left(R_{k}^{\prime}, Q\right), g_{k, \mid \partial R_{k}^{\prime}}=h_{k} \mid \partial R_{k}^{\prime}$ and by (13)

$$
\operatorname{Dir}\left(g_{k, i} ; R_{k}^{\prime} \sim R_{k}\right) \leqslant C_{8} \zeta^{\prime 2 m}\left(R_{k}\right) \text {, }
$$

whence

(16)

$$
\mathbf{G}\left(f_{i} ; R_{k}\right) \geqslant \mathbf{G}\left(g_{k, i} ; R_{k}^{\prime}\right)-C_{0} \operatorname{Dir}\left(g_{k, i} ; R_{k}^{\prime} \sim R_{k}\right) \geqslant \mathbf{G}\left(g_{k, i} ; R_{k}^{\prime}\right)-C_{9} \zeta L^{2} m\left(R_{k}\right) .
$$

Due to (2) and (3) we obtain from Lemma 4.6

$$
\begin{aligned}
\mathbf{G}\left(g_{k, i} ; R_{k}^{\prime}\right) \geqslant \mathbf{G}\left(h_{k} ; R_{k}^{\prime}\right)-C_{10}\left[\varepsilon \operatorname{Dir}\left(g_{k, i} ; R_{k}^{\prime}\right)+\varepsilon M^{2} L^{2 m}\left(R_{k}^{\prime}\right)\right. \\
\left.+M^{2} \mathbb{L}^{m}\left\{x \in R_{k}^{\prime}: \mathcal{G}\left(g_{k, i}(x), h_{k}(x)\right)>\delta\right\}\right] .
\end{aligned}
$$

Here

$$
\begin{gathered}
\mathbf{G}\left(h_{k} ; R_{k}^{\prime}\right) \geqslant \mathbf{G}\left(h_{k} ; R_{k}\right)-M^{2} \mathbb{L}^{m}\left(R_{k}^{\prime} \sim R_{k}\right) \geqslant \mathbf{G}\left(h_{k}, R_{k}\right)-\zeta L^{m}\left(R_{k}\right), \\
\varepsilon \operatorname{Dir}\left(g_{k, i} ; R_{k}^{\prime}\right) \leqslant \zeta \operatorname{Dir}\left(f_{i} ; R_{k}\right)+C_{8} \zeta^{m}\left(R_{k}\right), \\
\varepsilon M^{2} E^{m}\left(R_{k}^{\prime}\right)<2 \zeta L^{m}\left(R_{k}\right)
\end{gathered}
$$

and

$$
\begin{aligned}
M^{2} \mathbb{L}^{m} & \left\{x \in R_{k}^{\prime}: \dot{S}\left(g_{k, i}(x), h_{k}(x)\right)>\delta\right\} \\
& \leqslant M^{2}\left[\mathscr{L}^{m}\left(R_{k}^{\prime} \sim R_{k}\right)+\mathscr{L}^{m}\left\{x \in R_{k}: \mathcal{G}\left(f_{i}(x), h_{k}(x)\right)>\delta\right\}\right] \\
& \leqslant \zeta \mathscr{L}^{m}\left(R_{k}\right)+M^{2} \mathcal{E}^{2 m}\left(R_{k}\right)+M^{2} \mathbb{L}^{m}\left\{x \in R_{k}: \Theta\left(f_{i}(x), f(x)\right)>\delta / 2\right\} \\
& \leqslant 3 \zeta \mathscr{L}^{m}\left(R_{k}\right)
\end{aligned}
$$

for large $i$ by (8) and the fact that $\mathbb{L}^{m}\left\{x \in R_{k}: \mathcal{G}\left(f_{i}(x), f(x)\right)>\delta / 2\right\} \rightarrow 0$ as $i \rightarrow \infty$. Substituting these estimates in (17) we get

$$
\mathbf{G}\left(g_{k, i} ; R_{k}^{\prime}\right) \geqslant \mathbf{G}\left(h_{k} ; R_{k}\right)-C_{11}\left[\zeta \operatorname{Dir}\left(f_{i} ; R_{k}\right)+\zeta L^{m}\left(R_{k}\right)\right],
$$

which combined with (16) gives for large $i$

$$
\mathbf{G}\left(f_{i} ; R_{k}\right) \geqslant \mathbf{G}\left(h_{k} ; R_{k}\right)-C_{12}\left[\zeta \operatorname{Dir}\left(f_{i} ; R_{k}\right)+\zeta E^{m}\left(R_{k}\right)\right] \text {. }
$$


Summing over $k$ and recalling (15), (11) and (1) we get

$$
\begin{aligned}
\mathbf{G}\left(f_{i} ; R\right)= & \sum_{k=1}^{p} \mathbf{G}\left(f_{i} ; R_{k}\right)+\sum_{k=p+1}^{q} G\left(f_{i} ; R_{k}\right) \\
\geqslant & \sum_{k=1}^{p} G\left(h_{k} ; R_{k}\right)-C_{12}\left[\zeta \operatorname{Dir}\left(f_{i} ; R\right)+\zeta L^{m}(R)\right] \\
& -C_{3} \operatorname{Dir}\left(f ; \bigcup_{k=p+1}^{q} R_{k}\right)-C_{4} \zeta \varrho^{m}(R) \\
\geqslant & \mathbf{G}(f ; R)-C_{13} \zeta .
\end{aligned}
$$

Letting $i \rightarrow \infty$ and $\zeta \rightarrow 0$, we complete the proof.

4.8. Corollary. Suppose $A \subset R^{m}$ is bounded and open with $\mathfrak{H}^{m-1}(\partial A)<\infty$, $f, f_{i} \in \mathcal{O}_{2}(A, \mathbf{Q})$ and $f_{\mid \partial A}=f_{i \mid \partial A} \in \partial \mathcal{O}_{2}(\partial A, \mathbf{Q})$. If $\lim _{i \rightarrow \infty} \int_{A} \mathcal{G}\left(f_{i}, f\right)^{2} d \mathscr{L}^{m}=0$ and $\sup _{i} \operatorname{Dir}\left(f_{i} ; A\right)<\infty$, then $\mathbf{G}(f ; A) \leqslant \liminf _{i \rightarrow \infty} G\left(f_{i} ; A\right)$.

Proof. By 1.3(5) we can extend $f$ and $f_{i}$ to an open interval $R$ containing $A$ so that $f(x)=f_{i}(x)$ for $x \in R \sim A$ and $f, f_{i} \in \mathcal{O}_{2}(R, \mathbf{Q})$. Then the result follows from Theorem 4.7.

5. Existence of $\mathbf{G}$ minimizing $\mathbf{Q}$ valued functions. We call a function $f \in \mathcal{P}_{2}(A, \mathbf{Q})$ $\mathbf{G}$ minimizing if $\mathbf{G}(f ; A) \leqslant \mathbf{G}(g ; A)$ for all $g \in \mathcal{Y}_{2}(A, \mathbf{Q})$ with $g_{\mid \partial A}=f_{\mid \partial A}$.

5.1. Theorem. Suppose $A \subset R^{m}$ is bounded and open, $\partial A$ is $\left(\mathcal{H}^{m-1}, m-1\right)$ rectifiable, $G$ is a $Q$ elliptic integrand and $g \in \partial \mathcal{Y}_{2}(\partial A, \mathbf{Q})$. Then there is a $\mathbf{G}$ minimizing function $f \in \mathcal{Y}_{2}(A, \mathbf{Q})$ with $f_{\mid \partial A}=g$.

Proof. There are an open interval $R, A \subset R \subset \mathbf{R}^{m}$, and $\tilde{g} \in \mathcal{Y}_{2}(R, \mathbf{Q})$ with $\tilde{g}_{\mid \partial A}=g$ and $\tilde{g}_{\mid \partial R}=0$. If $f \in \mathscr{Y}_{2}(A, \mathbf{Q})$ with $f_{\mid \partial A}=g$, then we can extend $f$ to $R$ so that $f(x)=\tilde{g}(x)$ for $x \in R \sim A$ and $f \in \mathcal{Y}_{2}(R, \mathbf{Q})$. Let $c>0$ be a $Q$ ellipticity bound for $G$. Then, since $f_{\mid \partial R}=0$,

$$
c \operatorname{Dir}(f ; A) \leqslant c \operatorname{Dir}(f ; R) \leqslant \mathbf{G}(f ; R)=\mathbf{G}(f ; A)+G(\tilde{g} ; R \sim A) .
$$

Consequently, if $f_{i} \in \mathcal{Y}_{2}(A, \mathbf{Q})$ with $f_{i \mid \partial A}=g$ and

$$
\lim _{i \rightarrow \infty} \mathbf{G}\left(f_{i} ; A\right)=\lambda=\inf \left\{\mathbf{G}(h ; A): h \in \mathcal{O}_{2}(A, \mathbf{Q}), h_{\mid \partial A}=g\right\},
$$

then $\sup _{i} \operatorname{Dir}\left(f_{i} ; A\right)<\infty$, and thus by $[\mathbf{A}, A .1 .2(10)]$ there are a subsequence $\left(f_{i_{i}}\right)$ of $\left(f_{i}\right)$ and $f \in \mathscr{Y}_{2}(A, \mathbf{Q})$ such that $\lim _{j \rightarrow \infty} \int_{A} \mathcal{S}\left(f_{i}, f\right)^{2} d \mathcal{L}^{m}=0$, and, by $1.3(8), f_{\mid \partial A}=g$. From Corollary 4.8 we obtain

$$
\lambda \leqslant \mathbf{G}(f ; A) \leqslant \liminf _{j \rightarrow \infty} \mathbf{G}\left(f_{i} ; A\right)=\lambda,
$$

which proves the theorem.

6. Regularity of $\mathbf{G}$ minimizing functions on 2-dimensional domains. In this section we assume that $G$ is a $Q$ elliptic integrand with $Q$ ellipticity bound $c>0$. We shall prove that in the case $m=2$ the $\mathbf{G}$ minimizing functions are Hölder continuous and regular on an open dense set. 
6.1. Elementary facts about $Q$ elliptic integrands. Let $J$ be the set of those quadruples of integers $(i, j, k, l)$ for which $i, j \in\{1, \ldots, m\}, k, l \in\{1, \ldots, n\}$ and either $i \neq j$ or $k \neq l$. We associate with $G$ the quadratic integrands $G_{0}$ and $G_{i, j, k, l}$ for $(i, j, k, l) \in J$ such that for $p=\left(p_{i}^{k}\right)$

$$
G_{0}(p)=\sum_{i, k} q(i, i, k, k) p_{i}^{k^{2}}, \quad G_{i, j, k, l}(p)=q(i, j, k, l) p_{i}^{k} p_{j}^{l} .
$$

Then for any $f \in \mathrm{P}_{2}(A, \mathbf{Q})$

$$
\mathbf{G}(f ; A)=\mathbf{G}_{0}(f ; A)+\sum_{J} \mathbf{G}_{i, j . k . l}(f ; A) .
$$

Since $G$ satisfies the strong ellipticity condition (cf. Introduction), it follows that $q(i, i, k, k)>0$ for all $i, k$. Hence there is a positive number $a$ such that

$$
a|p|^{2} \leqslant G_{0}(p) \text {. }
$$

The set of $Q$ elliptic integrands is open:

(2) Lemma. Suppose $0<\delta<c /(m n)$ and $G^{\prime}$ is a quadratic integrand, $G^{\prime}(p)=$ $\Sigma q^{\prime}(i, j, k, l) p_{i}^{k} p_{j}^{l}$, such that $\left|q^{\prime}(i, j, k, l)-q(i, j, k, l)\right| \leqslant \delta$ for all $i, j, k, l$. Then $G^{\prime}$ is $Q$ elliptic with $Q$ ellipticity bound $c-m n \delta$.

Proof. Schwarz's inequality gives $\left|G^{\prime}(p)-G(p)\right| \leqslant m n \delta|p|^{2}$, which implies $G^{\prime}(p) \geqslant G(p)-m n \delta|p|^{2}$, and the lemma follows from the definition of $Q$ ellipticity.

Next we give a generalization of Lemma 4.4.

6.2. LEMMA. There is a positive number $b$ depending only on $m, n, Q$ and $G$ such that whenever $U$ is an open ball in $R^{m}, f \in \mathrm{G}_{2}(U, \mathbf{Q}), \eta \in \mathbf{Q}$ and $f_{\mid \partial U}=\eta$, then $b \operatorname{Dir}(f ; U)$ $\leqslant \mathbf{G}(f ; U)$.

Proof. By homogeneity we may assume $U$ is the unit ball. If the lemma is false there are sequences $\eta_{\nu} \in \mathbf{Q}$ and $f_{\nu} \in \mathrm{Q}_{2}(U, \mathbf{Q})$ with $f_{\nu \mid \partial U}=\eta_{\nu}, \nu=1,2, \ldots$, such that $\operatorname{Dir}\left(f_{\nu} ; U\right)=1$ and $\mathbf{G}\left(f_{\nu} ; U\right)<1 / \nu$. Let $a$ be as in $6.1(1)$ and $\delta$ as in 6.1(2), $\varepsilon=\delta / \max \{q(i, j, k, l)\}$, and let $\nu>\max \left\{2 m^{2} n^{2} /(a \varepsilon), 2 / a\right\}$. Then

$$
\sum_{J} \mathbf{G}_{i, j, k, l}\left(f_{\nu} ; U\right)=\mathbf{G}\left(f_{\nu} ; U\right)-\mathbf{G}_{0}\left(f_{\nu} ; U\right)<1 / \nu-a \operatorname{Dir}\left(f_{\nu} ; U\right)<-a / 2 \text {. }
$$

As card $J<m^{2} n^{2}$, there is $(i, j, k, l) \in J$ for which

$$
\mathbf{G}_{i, j, k, l}\left(f_{\nu} ; U\right)<-a /\left(2 m^{2} n^{2}\right) .
$$

Define the integrand $G^{\prime}$ by replacing $q(i, j, k, l)$ by $(1+\varepsilon) q(i, j, k, l)$ and keeping the other coefficients of $G$ unchanged. Then 6.1(2) implies $G^{\prime}$ is $Q$ elliptic. But on the other hand

$$
\mathbf{G}^{\prime}\left(f_{\nu} ; U\right)=\mathbf{G}\left(f_{\nu} ; U\right)+\varepsilon \mathbf{G}_{i, j . k . l}\left(f_{\nu} ; U\right)<1 / \nu-\varepsilon a /\left(2 m^{2} n^{2}\right)<0,
$$

which contradicts Lemma 4.4 .

We denote by $U^{m}(z, r)$ and $B^{m}(z, r)$ the open and closed balls in $R^{m}$ with centre $z$ and radius $r$. 
6.3. Lemma. There are positive numbers $C$ and $\omega$ depending only on $m, n, Q$ and $G$ with the following property:

If $f \in \mathrm{S}_{2}\left(U^{m}(0,1), \mathbf{Q}\right)$ is strictly defined and $\mathbf{G}$ minimizing and $z \in U^{m}(0,1)$, then for all $0<r<1-|z|, 0<s \leqslant 1$,

(1) $\operatorname{Dir}\left(f ; B^{m}(z, r)\right) \leqslant C r \operatorname{dir}\left(f ; \partial B^{m}(z, r)\right)$,

(2) $\operatorname{Dir}\left(f ; B^{m}(z, s r)\right) \leqslant C s^{\omega} \operatorname{Dir}\left(f ; B^{m}(z, r)\right)$.

Proof. Let $g \in{ }^{\prime \prime}{ }_{2}\left(U^{m}(z, r), \mathbf{Q}\right)$ be Dirichlet minimizing with $g_{\mid \partial B^{m}(z, r)}=f_{\mid \partial B^{\prime \prime}(z, r)}$. It follows from [A, 2.7(2)(i), (ii) and 2.12] that

$$
\operatorname{Dir}\left(g ; B^{m}(z, r)\right) \leqslant C_{1} r \operatorname{dir}\left(f ; \partial B^{m}(z, r)\right),
$$

where $C_{1}$ depends only on $m, n$, and $Q$. As in the proof of Lemma 4.5 we can employ the Poincaré inequality to find $h \in \mathcal{O}_{2}\left(U^{m}(0,1), \mathbf{Q}\right)$ such that $h \mid B^{m}(z, r)=$ $g \mid B^{m}(z, r), h_{\partial B^{m}(z, 2 r)}=\eta$ for some $\eta \in \mathbf{Q}$ and

$\operatorname{Dir}\left(h ; B^{m}(z, 2 r) \sim B^{m}(z, r)\right) \leqslant C_{2} \operatorname{Dir}\left(g ; B^{m}(z, r)\right) \leqslant C_{1} C_{2} r \operatorname{dir}\left(f ; \partial B^{m}(z, r)\right)$.

Defining $\tilde{h} \in \mathcal{O}_{2}\left(U^{m}(0,1), \mathbf{Q}\right)$ by $\tilde{h}(x)=f(x)$ for $x \in B^{m}(z, r)$ and $\tilde{h}(x)=h(x)$ otherwise, we have by Lemma 6.2

$$
\begin{aligned}
b \operatorname{Dir}\left(f ; B^{m}(z, r)\right) & \leqslant b \operatorname{Dir}\left(\tilde{h} ; B^{m}(z, 2 r)\right) \\
& \leqslant \mathbf{G}\left(\tilde{h} ; B^{m}(z, 2 r)\right) \\
& =\mathbf{G}\left(f ; B^{m}(z, r)\right)+\mathbf{G}\left(h ; B^{m}(z, 2 r) \sim B^{m}(z, r)\right) \\
& \leqslant \mathbf{G}\left(g ; B^{m}(z, r)\right)+\mathbf{G}\left(h ; B^{m}(z, 2 r) \sim B^{m}(z, r)\right) \\
& \leqslant C_{0} \operatorname{Dir}\left(g ; B^{m}(z, r)\right)+C_{0} \operatorname{Dir}\left(h ; B^{m}(z, 2 r) \sim B^{m}(z, r)\right) \\
& \leqslant C_{0} C_{1}\left(1+C_{2}\right) r \operatorname{dir}\left(f ; \partial B^{m}(z, r)\right) .
\end{aligned}
$$

This proves (1). Using the fact that the function $\phi, \phi(r)=\operatorname{Dir}\left(f ; B^{m}(z, r)\right)$, is absolutely continuous with $\phi^{\prime}(r) \geqslant \operatorname{dir}\left(f ; \partial B^{m}(z, r)\right)$ for $L^{l}$ almost all $0<r<1-$ $|z|$, one derives (2) from (1) by integration (cf. [A, 2.13(2)]).

6.4. THEOREM. There are positive numbers $C$ and $\omega$ depending only on $m, n, Q$ and $G$ with the following property:

If $f \in \mathscr{S}_{2}\left(U^{2}(0,1), \mathbf{Q}\right)$ is strictly defined and $\mathbf{G}$ minimizing, $0<\delta<1$ and $x, y \in$ $B^{2}(0,1-\delta)$, then

$$
\mathcal{S}(f(x), f(y)) \leqslant C \delta^{-1} \operatorname{Dir}\left(f ; B^{2}(0,1)\right)^{1 / 2}|x-y|^{\omega} .
$$

In particular, $f \mid B^{2}(0,1-\delta)$ is Hölder continuous with exponent $\omega$.

This follows from 6.3(2) as [A, 2.13(3)], see also [M, Theorem 3.5.2].

6.5. Theorem. Suppose $f \in \mathcal{O}_{2}\left(U^{m}(0,1), \mathbf{Q}\right)$ is continuous and $\mathbf{G}$ minimizing. Define $\sigma: U^{m}(0,1) \rightarrow\{1,2, \ldots, Q\}$

$\sigma(x)=$ card spt $f(x)$ for $x \in U^{m}(0,1)$ (recall $f(x)$ is a measure $)$,

$V=\left\{x \in U^{m}(0,1): \sigma\right.$ is continuous at $\left.x\right\}$. 
Then $V$ is open and dense in $U^{m}(0,1)$. Furthermore, for every $x \in V$ there are $a$ neighborhood $W$ of $x$ and real analytic functions $f_{1}, \ldots, f_{Q}: W \rightarrow R^{n}$ such that

$$
f(y)=\sum_{i=1}^{Q}\left[\left[f_{i}(y)\right]\right] \quad \text { for } y \in W .
$$

Proof. Using the continuity of $f$ one easily verifies that $V$ is open and dense, and that for every $x \in V$ there are a neighborhood $W$ of $x$ and continuous functions $f_{1}, \ldots, f_{Q} \in v_{2}\left(W, R^{n}\right)$ such that $f(y)=\sum_{i=1}^{Q}\left[\left[f_{i}(y)\right]\right]$ for $y \in W$. Then each $f_{i}$ is $\mathbf{G}$ minimizing (cf. [A, 2.14.10(3)]), and hence real analytic by [M, §6.6].

\section{REFERENCES}

[A] F. J. Almgren. Jr., Q valued functions minimizing Dirichlet's integral and the regularity of area minimizing integral currents up to codimension two, preprint.

[A1] E_ Existence and regularity almost everyhere of solutions to elliptic variational problems among surfaces of varying topological type and singularity structure. Ann. of Math. (2) 87 (1968), $321-391$.

[A2] _. Dirichlet's problem for multiple valued functions and the regularity of mass minimizing integral currents, Minimal Submanifolds and Geodesics (M. Obata, editor), Kaigai, Tokyo, 1978, pp. 1-6.

[F] H. Federer, Geometric measure theorl. Springer-Verlag. Berlin and New York, 1969.

[GT] D. Gilbarg and N. S. Trudinger. Elliptic partial differential equations of second order. SpringerVerlag, Berlin and New York, 1977.

[H] L. van Hove, Sur l'extension de la condition de Legendre du calcul des cariations aux intégrales multiples à plusieurs fonctions inconnues, Indag. Math. 9 (1947), 3-8.

[M] C. B. Morrey. Jr., Multiple integrals in the calculus of variations, Springer-Verlag. Berlin and New York. 1966.

[S] V. Scheffer, Regularity and irregularity of solutions to non-linear second order elliptic sistems of partial differential equations and inequalities, Princeton Univ. Thesis, Princeton, N.J., 1974.

[SE] E. M. Stein. Singular integrals and differentiability properties of functions, Princeton Univ. Press. Princeton. N.J.. 1970.

[T] F. J. Terpsta, Die Darstellung biquadratischer Formen als Summen con Quadraten mit Anwendung auf die Variationsrechmung. Math. Ann. 116 (1938), 166-180.

Department of Mathematics, University of Helsinki, Helsinki, Finland 